\title{
HARMONIC FUNCTIONS ON OPEN RIEMANN SURFACES
}

\author{
BY \\ H. L. ROYDEN( $\left.{ }^{1}\right)$
}

In recent years the importance of open (noncompact) Riemann surfaces for function theory has been shown by the work of Nevanlinna, Ahlfors, and Myrberg. In particular there arose the concept of the type of a Riemann surface: Nevanlinna considered Riemann surfaces whose boundary has zero harmonic measure [20], and Myrberg the existence of a Green's function [15]. Elegant results concerning the type of a covering surface were given by Ahlfors [1]. An extension of this idea of type leads to the concept of the classification of Riemann surfaces as suggested by Ahlfors and Sario [21]. The modern theory of open Riemann surfaces has been vigorously developed along these lines not only by these but also by the younger members of the Finnish school: Sario, Virtanen, Laasonen, and Lehto.

It is the purpose of the present paper to consider in some detail the family of harmonic functions on such a Riemann surface and to apply this study to questions concerning the type and classification problems.

In the first two chapters are collected a number of results which are necessary for the later chapters. We have put these results in just that form in which they are needed here, but have omitted proofs in general, since they require only minor modifications from proofs given in the literature.

In the third chapter we consider the structure of the space $B D$ of functions on a Riemann surface which are both bounded and have a finite Dirichlet integral. We introduce a convergence topology into this space and consider some of the linear functionals continuous in this topology. The class of functions which vanish outside a compact set is denoted by $K$, and its closure in $B D$ denoted by $\bar{K}$. We then show that for every $f \in B D$ we have

$$
f=f_{K}+u,
$$

where $f_{K} \in \bar{K}$, and $u \in H B D$, i.e., is a harmonic $B D$ function. The decomposition is unique except for those Riemann surfaces for which

$$
\bar{K}=B D .
$$

We call these surfaces parabolic and show that they have a null boundary in the sense of Nevanlinna and have no Green's function. Conversely, if a surface has a boundary which has zero harmonic measure in the sense of Nevan-

Received by the editors September 24, 1951.

(1) The results of this paper are contained in the author's thesis at Harvard University, April, 1951. 
linna, or if it does not have a Green's function, then it is parabolic.

We also give a criterion in terms of the continuous linear functionals on the space $B D$ that a Riemann surface be hyperbolic (Theorem 4).

In the fourth chapter we extend these results to the case of surfaces with a relative boundary and show that if $f \in B D$, then

$$
f=f_{0}+u_{K}+u_{N},
$$

where $f_{0} \in \bar{K} O$, that is, belongs to $\bar{K}$ and vanishes on the relative boundary $R$ of the Riemann surface, $u_{K} \in H \bar{K}$, that is, belongs to $\bar{K}$ and is harmonic, while $u_{N} \in H N$, that is, it is an $H B D$ function whose normal derivative vanishes on $R$. These composition theorems correspond roughly to the classical Dirichlet and Neumann problems. As a consequence it is readily proved that there is in this case a norm preserving isomorphism between the classes $H O$ and $H N$, where the norm of a function is defined as the supremum of its absolute value.

If an open Riemann surface $W$ is separated by a finite number of compact curves into a finite number of surfaces bounded (relatively) by some of these curves, then we show that the space of $H B D$ functions on $W$ is isomorphic to the linear direct sum of the spaces $H O$ (or equivalently $H N$ ) on the bounded surfaces.

In the last chapter we again return to the type problem and obtain some conditions in terms of a triangulation on a Riemann surface that the surface be hyperbolic. The results in this chapter are somewhat similar in form to those of Wittich, but are complementary to his in that we give here sufficient conditions that a Riemann surface be hyperbolic, while he considers necessary conditions.

Much of the background for this paper is to be found in the works of Ahlfors and Nevanlinna in general and in particular in the seminar on open Riemann surfaces given by the former at Harvard in the fall of 1949. I owe much to Professor Lars Ahlfors for his valuable help and kindly encouragement in this investigation.

\section{Chapter I. RiemanN SURFACES: PREliminaRY CONCEPTS}

1. Definitions. A Riemann surface $W$ is given by a connected, separable Hausdorff space and a covering $\{U\}$ by open sets together with a collection $\{z\}$ of corresponding homeomorphisms onto open sets in the complex z-plane with the property that whenever two open sets $U_{1}$ and $U_{2}$ meet, then $z_{2} \circ z_{1}^{-1}$ is a complex analytic function in the region $z_{1}\left(U_{1} \cap U_{2}\right)$. The variable $z_{1}$, which can be used to label points in the neighborhood $U_{1}$, is said to be a local uniformizer valid for the region $U_{1}$ or more briefly a uniformizer for $U_{1}$. We shall use the letter $W$ to denote both the Riemann surface and the underlying topological space.

We say that a mapping 


$$
F: \quad W_{1} \rightarrow W_{2}
$$

of one Riemann surface into another is analytic at a point $p \in W_{1}$ if for a uniformizer $z_{1}$ valid near $p$ and a uniformizer $z_{2}$ valid near $F(p)$ the function $z_{2} \circ F \circ z_{1}^{-1}$ is a complex analytic function near $z_{1}^{-1}(p)$. If $F$ is a one-to-one analytic mapping of $W_{1}$ into $W_{2}$, we shall call it a conformal mapping into. A conformal mapping is clearly a homeomorphism into. By an analytic arc we mean the conformal image of a closed interval in the complex plane.

By a compact region we shall mean an open connected set whose boundary consists of a finite number of piecewise analytic curves. It is readily shown that any compact set on a Riemann surface can be included in the interior of a compact region.

A sequence $\left\{\Omega_{i}\right\}$ of compact regions with $\bar{\Omega}_{i} \subset \Omega_{i+1}$ and $U \Omega_{i}=W$ will be termed an exhaustion of $W$. The existence of an exhaustion of an open (i.e., noncompact) Riemann surface $W$ is a simple consequence of the separability of $W$.

A sequence $\left\{\Omega_{i}\right\}$ of disjoint compact regions with $U \bar{\Omega}_{i}=W$ is called a subdivision of $W$ provided that a point on the boundary of a region $\Omega_{i}$ which is not a "corner" belongs to the boundary of exactly one other region, and provided also that a given compact set on the Riemann surface meets only a finite number of the $\Omega_{i}$. We shall usually assume that the compact regions $\Omega_{i}$ are so small that we can find uniformizers $z_{i}$ such that each $z_{i}$ is valid in some open set containing $\bar{\Omega}_{i}$. The existence of such a subdivision is immediate.

Let $V$ be a connected, separable Hausdorff space and $W$ a dense open subset of $V$. Then we shall say that $V$ is a bounded Riemann surface with interior $W$ and boundary $R=V-W$ provided there is a covering $\{U\}$ of $V$ by open sets and a collection of corresponding homeomorphisms $\{z\}$ into the complex $z$-plane such that $z(U \cap W)$ is an open set of the complex $z$-plane, $z$ is real on $U \cap R$, and whenever $U_{1}$ and $U_{2}$ meet, we have $z_{2} \circ z_{1}{ }^{-1}$ a complex analytic function on $z_{1}\left(U_{1} \cap U_{2}\right)$. We shall use the letter $V$ to denote both the bounded Riemann surface and the underlying topological space. It should be noted that the interior of a bounded Riemann surface is a Riemann surface and that a Riemann surface may be considered to be a bounded Riemann surface with an empty boundary.

The notions of analytic and conformal mappings extend easily to bounded Riemann surfaces.

An important example of a bounded Riemann surface is that obtained by taking $V=\bar{\Omega}$, where $\Omega$ is a compact region on a Riemann surface $W^{*}$, and setting $W=\Omega$ with a uniformizer for $U \subset W$ defined to be the corresponding uniformizer on $W^{*}$. Uniformizers can then be constructed for points belonging to the boundary of $\Omega$. It should be noted that the class of functions analytic on $V$ may differ from the class of functions analytic on $\bar{\Omega}$ in that different requirements are imposed at the corners of $\Omega$.

Remembering that a continuum of the boundary of a bounded Riemann 
surface is an analytic arc, we define a compact region on $V$ to be a connected open set whose closure in $V$ is compact and whose boundary is composed of a finite number of piecewise analytic curves. By an exhaustion of $V$ we shall mean a sequence $\left\{\Omega_{i}\right\}$ of compact regions such that $\bar{\Omega}_{i} \subset \Omega_{i+1} \cup R$, while $W=\mathrm{U} \Omega_{i}$ and $V=\mathrm{U} \bar{\Omega}_{i}$. A sequence $\left\{\Omega_{i}\right\}$ of disjoint compact regions is to be called a subdivision of $V$ provided that $V=U \bar{\Omega}_{i}$, and that a point other than a corner which belongs to the boundary of some $\Omega_{i}$ belongs either to $R$ or to the boundary of exactly one other compact region. It is to be noted that an exhaustion or subdivision of a bounded Riemann surface is not an exhaustion or subdivision of its interior unless the bounded Riemann surface has an empty boundary.

If $V$ is a bounded Riemann surface, it is well known that $V$ can be extended by a process of symmetrization to a Riemann surface $V^{\wedge}$ called the double of $V$. There exists an involutary, indirectly conformal mapping of $V^{-}$ onto itself such that every point of the boundary $R$ of $V$ remains fixed. The image of $p \in W$ is denoted by $\tilde{p}$, the image of $W$ by $\tilde{W}$.

A function $f$ on $V^{\wedge}$ is called symmetric if $f(\widetilde{p})=f(p)$, while it is called skew-symmetric if $f(\tilde{p})=-f(p)$. If $f$ is an arbitrary function on $V^{\frown}$, we shall call the function $f_{s}=[f(p)+f(\tilde{p})] / 2$ the symmetrization of $f$. If $f$ is defined and continuous on $V$, then there is a unique symmetric function on $V^{-}$ which is equal to $f$ on $V$. This function is continuous and is called the symmetric extension of $f$ to $V^{\frown}$. Similarly, if $f$ is defined and continuous on $V$ and has the value zero on the boundary of $V$, then there is a unique skewsymmetric function defined and continuous on $V^{-}$and equal to $f$ on $V$. This function is called the skew-symmetric extension of $f$.

2. Harmonic functions and Harnack's theorem. A function $u$ defined on a Riemann surface is said to be harmonic in a region if at each point of the region it is a harmonic function of a uniformizer at that point. The definition is easily seen to be independent of the uniformizers chosen. We shall have considerable use for the following lemma concerning harmonic functions:

LEMma 1 (HARNACK'S THEOREM). Let $\left\{u_{i}\right\}$ be a sequence of positive functions on a Riemann surface $W$ with the property that, given any compact region $\Omega$ on $W$, all $u_{i}$ are harmonic in $\Omega$ from some $i$ on. Then either $\left\{u_{i}\right\}$ contains $a$ subsequence which converges to a function $u$ harmonic on $W$, the convergence being uniform on each compact region of $W$, or else the sequence $\left\{u_{i}\right\}$ diverges to $+\infty$ uniformly on every compact set.

The proof is almost identical with the proof of the usual form of Harnack's theorem in the plane and is left to the reader. As an immediate consequence we have the fact that a uniformly bounded sequence of functions which are ultimately harmonic in every compact region must possess a subsequence which converges uniformly on every compact set to a harmonic function.

Let $\Omega$ be a compact region on a Riemann surface $W$. We shall say that the 
derivatives of a sequence $\left\{f_{i}\right\}$ converge to the derivatives of $f$, uniformly in the compact region $\Omega$, if there is a finite set $\left\{U_{k}\right\}$ of neighborhoods covering $\Omega$ with the property that for each $U_{k}$ there is a uniformizer $z_{k}$ valid in it such that the derivatives of the functions $f_{i}$ with respect to $x_{k}$ and $y_{k}\left(z_{k}=x_{k}+i y_{k}\right)$ converge to the derivatives of $f$ with respect to $x_{k}$ and $y_{k}$ uniformly in the neighborhood $U_{k}$. The following lemma is an easy consequence of this definition.

LEMMA 2. Let $\left\{u_{i}\right\}$ be a sequence of functions on a Riemann surface $W$ with the property that given any compact region $\Omega$ on $W$ all $u_{i}$ are harmonic in $\Omega$ from some $i$ on, and suppose that $u_{i} \rightarrow u$ uniformly on every compact region. Then $u$ is harmonic and the derivatives of $\left\{u_{i}\right\}$ converge to those of $u$ uniformly on every compact region.

3. Differentials. A first order differential on a Riemann surface $W$ is an expression of the form

$$
\alpha=a d x+b d y
$$

where $a$ and $b$ are complex-valued functions which depend on the uniformizer $z=x+i y$ in such a way that $\alpha$ is independent of the uniformizer chosen.

A second order differential is an expression

$$
\mu=c d x d y,
$$

where again $c$ depends on the uniformizer in such a way that the differential is independent of the choice of the uniformizer.

The sum of two differentials of the same order and the product of a function and a differential are defined in the obvious way. The product of two first order differentials $\alpha_{1}=a_{1} d x+b_{1} d y$ and $\alpha_{2}=a_{2} d x+b_{2} d y$ is by definition

$$
\alpha_{1} \alpha_{2}=\left(a_{1} b_{2}-a_{2} b_{1}\right) d x d y,
$$

which is easily seen to be independent of the choice of the uniformizer and anti-commutative.

If $f$ is a function whose partial derivatives exist in some sense, we define the differential of $f$ to be

$$
d f=\frac{\partial f}{\partial x} d x+\frac{\partial f}{\partial y} d y,
$$

and define the differential of a first order differential as

$$
d \alpha=\left(\frac{\partial b}{\partial x}-\frac{\partial a}{\partial y}\right) d x d y .
$$

It is easily verified that these definitions are invariant with respect to changes of uniformizer. We have the important relations 


$$
d(d f)=0
$$

and

$$
d(f \alpha)=d f \alpha+f d \alpha .
$$

In view of the analytic structure of our Riemann surface we may define for each first order differential $\alpha=a d x+b d y$ a dual differential

$$
* \alpha=-b d x+a d y \text {. }
$$

The invariance of this operator is a simple consequence of the Cauchy-Riemann differential equations which hold between different uniformizers.

We have the following identities involving this operation:

$$
* * \alpha=-\alpha, \quad \alpha_{1} * \alpha_{2}=\alpha_{2} * \alpha_{1}, \quad \alpha * \bar{\alpha}=\left(|a|^{2}+|b|^{2}\right) d x d y,
$$

where we have written $\bar{\alpha}=\bar{a} d x+\bar{b} d y$ for the conjugate of the differential $\alpha$.

We say that a function $f$ is of class $C^{k}$ on a region if $f$ together with its first $k$ derivatives are defined and continuous there. We say that a function is of class $C^{\infty}$ if it has continuous derivatives of all orders. A differential is said to be of class $C^{k}$ if it has coefficients which are of class $C^{k}$.

We say that a differential $\alpha$ vanishes or is zero at a point $p_{0}$ if $a\left(p_{0}\right)$ $=b\left(p_{0}\right)=0$. Let $J$ be an analytic arc, and let $z$ be a uniformizer at a point $p_{0} \in J$ chosen so that $z$ is real on $J$. Then $\alpha=a d x+b d y$ is said to vanish along $J$ at $p_{0}$ if $a\left(p_{0}\right)=0$. Two first order differentials are said to be equal along an analytic arc $J$ if their difference vanishes along $J$.

The carrier (French: support) of a function or differential is the closure of the set of points where the function or differential is not zero.

Let $F$ be an analytic function which maps a Riemann surface $W_{1}$ into $W_{2}$. Then the adjoint mapping $F^{*}$ carries functions on $W_{2}$ into functions on $W_{1}$, and we may extend its definition so that it will carry differentials on $W_{2}$ into differentials on $W_{1}$ by noting that $F^{*}$ is linear and requiring that

$$
d F^{*} f=F^{*} d f
$$

For further details the reader may consult the excellent treatment given in [11].

Consider a second order differential

$$
\mu=c d x d y,
$$

and define the new differential

$$
|\mu|=|c| d x d y
$$

called the absolute value of $\mu$. Its definition is independent of the choice of uniformizer since all changes of uniformizer have a positive Jacobian. We say that $\mu$ is locally integrable if every point has a neighborhood $U$ in which 


$$
\iint_{U} c d x d y
$$

exists in the sense of Lebesgue.

If $\mu$ is locally integrable on $W$, we can define

$$
\iint_{W}|\mu|
$$

in the usual manner. We say that $\mu$ is integrable over $W$ if

$$
\iint_{W}|\mu|<\infty
$$

In this case it is possible to define

$$
\iint_{w}^{\mu}
$$

and we have

$$
\left|\iint_{W} \mu\right| \leqq \iint_{W}|\mu|
$$

as well as the usual Lebesgue convergence theorems.

A first order differential $\alpha$ is said to be locally square integrable if $\alpha * \bar{\alpha}$ is locally integrable. We shall call $\alpha$ square integrable (on $W$ ) if $\alpha * \bar{\alpha}$ is integrable there. The Schwarz inequality

$$
\left\{\iint\left|\alpha_{1} \alpha_{2}\right|\right\}^{2} \leqq \iint \alpha_{1} * \bar{\alpha}_{1} \iint \alpha_{2} * \bar{\alpha}_{2}
$$

is easily established as well as the fact that $\alpha_{1} \alpha_{2}$ is integrable whenever the right-hand side is finite.

We say that a property holds almost everywhere on a Riemann surface if it holds on a set $E$ which has the property that in any region for which there is a uniformizer $z$ valid $z$ maps $E$ into a set of measure zero in the $z$-plane. Henceforth, we shall always assume that first order differentials are defined almost everywhere and are locally square integrable. It is also assumed that second order differentials are defined almost everywhere and are locally integrable.

If $J$ is a piecewise analytic arc, and if $\alpha=a d x+b d y$ is a first order differential which is defined and continuous in some region containing $J$, we define

$$
\int_{J} \alpha=\int_{s_{1}}^{s_{2}}\left(a \frac{d x}{d s}+b \frac{d y}{d s}\right) d s
$$


where $s$ is some suitable parameter on $J$.

4. Continuity and Green's theorem. We shall say that a function $f$ is piecewise continuously differentiable or more briefly piecewise smooth on a Riemann surface $W$ if $f$ is continuous on $W$ and there is a subdivision $\left\{\Omega_{i}\right\}$ of $W$ such that $f \in C^{1}$ in each $\Omega_{i}$ and

$$
\iint_{\Omega} d f * d \bar{f}<\infty .
$$

It is also useful to consider a type of generalized continuity for differentials which we call g.-continuity for brevity: A first order differential $\alpha=a d x+b d y$ is said to be g.-continuous on a Riemann surface if there is a subdivision $\left\{\Omega_{i}\right\}$ such that

(i) At a corner $p$ of the subdivision there is a uniformizer $z$ such that

$$
|a(z)|^{2}+|b(z)|^{2}=O\left(|z-z(p)|^{-1}\right) .
$$

(ii) If $z$ is a uniformizer which is real on an edge of the subdivision, then $a(z)$ is continuous on the edge, except possibly at the corners.

(iii) In each $\Omega_{i}$ we have $\alpha \in C^{1}$, and

$$
\iint_{\Omega_{i}}|d \alpha|<\infty \text {. }
$$

For a bounded Riemann surface $V$ we require that $\left\{\Omega_{i}\right\}$ be a subdivision of $V$.

It should be noted that the sum as well as the product of two piecewise smooth functions is again piecewise smooth, while the sum of two g.-continuous differentials is again g.-continuous as well as the product of a g.-continuous differential by a piecewise smooth function. Some of the importance of this type of functions and differentials is illustrated by the following easily proved lemma:

LEMMA 3 (GREEN'S THEOREM). Let $\Omega$ be a compact region with the boundary $J$, and let $\alpha$ be a first order g.-continuous differential on $\Omega$. Then

$$
\int_{J} \alpha=\iint_{\Omega} d \alpha
$$

where the direction along $J$ is chosen so that $\Omega$ is always to the left.

Chapter II. Orthogonal projection and the spaCe $\Gamma$

5. Some basic properties. On an open Riemann surface $W$ we define the space $\Gamma=\Gamma(W)$ to consist of those first order differentials $\alpha$ for which

$$
\iint \alpha * \bar{\alpha}<\infty \text {. }
$$


In $\Gamma$ we define the norm $\|\alpha\|=\|\alpha\|_{w}$ of $\alpha$ by

$$
\|\alpha\|^{2}=\iint_{W} \alpha * \bar{\alpha} .
$$

The norm is a non-negative real number which is zero only if $\alpha$ vanishes almost everywhere. It has the following properties:

$$
\|\alpha\|=\|\bar{\alpha}\|=\|* \alpha\|=\|f \alpha\|
$$

provided $f$ is a function with $|f|=1$ almost everywhere. If we set

$$
(\alpha, \beta)=\iint \alpha * \bar{\beta},
$$

we have

$$
\begin{gathered}
\|\alpha\|^{2}=(\alpha, \alpha), \quad(\alpha, \beta)=(\beta, \alpha)^{-}, \\
(* \alpha, \beta)=-(\alpha, * \beta), \quad\left(\lambda_{1} \alpha_{1}+\lambda_{2} \alpha_{2}, \beta\right)=\lambda_{1}\left(\alpha_{1}, \beta\right)+\lambda_{2}\left(\alpha_{2}, \beta\right)
\end{gathered}
$$

where $\lambda_{1}$ and $\lambda_{2}$ are complex constants. Thus with $(\alpha, \beta)$ as a scalar product $\Gamma$ becomes an incomplete Hilbert space if we identify differentials which are equal almost everywhere. We have at once the Schwarz inequality

$$
|(\alpha, \beta)| \leqq\|\alpha\| \cdot\|\beta\|
$$

and the Minkowski inequality

$$
\|\alpha+\beta\| \leqq\|\alpha\|+\|\beta\| \text {. }
$$

It follows from the Riesz-Fischer theorem that $\Gamma$ is complete in this norm and hence is actually a Hilbert space. We say that a sequence $\alpha_{i} \in \Gamma$ converges, or converges strongly, if

$$
\left\|\alpha_{m}-\alpha_{n}\right\| \rightarrow 0
$$

as $m, n \rightarrow \infty$. The completeness of $\Gamma$ is then just the assertion that for such a sequence there is an $\alpha \in \Gamma$ such that

$$
\left\|\alpha_{m}-\alpha\right\| \rightarrow 0
$$

as $m \rightarrow \infty$. In this case we say that $\alpha_{i}$ converges to $\alpha$, and write

$$
\alpha_{i} \rightarrow \alpha \text {. }
$$

If for every $\beta \in \Gamma$

$$
\left(\alpha_{i}, \beta\right) \rightarrow(\alpha, \beta),
$$

then we say that $\alpha_{i}$ converges to $\beta$ weakly, and write

$$
\alpha_{i} \rightarrow \beta
$$


Clearly strong convergence implies weak convergence, but not vice versa. A set of elements of $\Gamma$ is said to be strongly closed if it contains all of its strong limit points, and weakly closed if it contains all of its weak limit points. Thus weak closure implies strong closure.

The following lemmas are well known theorems in the theory of Hilbert space [33].

Lemma 4. A linear subspace $A \subset \Gamma$ is weakly closed if and only if it is strongly closed.

Lemma 5. If $\alpha_{i} \rightarrow \alpha$ weakly, then $\|\alpha\| \leqq \lim$ inf $\left\|\alpha_{i}\right\|$.

LEMMA 6. If $\left\|\alpha_{n}\right\| \leqq 1$, then there is a subsequence $\alpha_{n_{i}}$ which converges weakly; i.e., the unit sphere is compact in the weak topology of $\Gamma$.

Lemma 7. If $\alpha_{i}$ converges, and if $\alpha_{i} \rightarrow \alpha$ weakly, then $\alpha_{i} \rightarrow \alpha$ strongly.

We shall also need the following lemma.

Lemma 8. Let $\alpha_{i}$ be a sequence of differentials in $\Gamma$ with $\left\|\alpha_{i}\right\|$ uniformly bounded, and suppose that there is a locally square integrable differential $\alpha$ such that for any compact region $\Omega \subset W$ and any $\beta \in \Gamma$ we have

$$
\iint_{\Omega} \alpha_{i} * \bar{\beta} \rightarrow \iint_{\Omega} \alpha * \bar{\beta}
$$

Then $\alpha \in \Gamma$, and $\alpha_{i} \rightarrow \alpha$ weakly.

Proof. Let $\alpha_{i_{k}}$ be an arbitrary subsequence of $\alpha_{i}$. By Lemma 6 there is a differential $\alpha_{0} \in \Gamma$ and a subsequence $\alpha_{i_{k_{j}}}$ which converges to $\alpha_{0}$ weakly. Let $\alpha_{\Omega}$ be equal to $\alpha_{0}-\alpha$ on $\Omega$ and vanish outside $\Omega$. Thus $\alpha_{\Omega} \in \Gamma$, and

$$
\iint \alpha * \bar{\alpha}_{\Omega}=\lim \iint_{\Omega} \alpha_{i_{k_{j}} * \bar{\alpha}_{\Omega}}=\iint \alpha_{0} * \bar{\alpha}_{\Omega} .
$$

Therefore

$$
\left\|\alpha-\alpha_{0}\right\|_{\Omega}^{2}=\iint\left(\alpha-\alpha_{0}\right) * \bar{\alpha}_{\Omega}=0,
$$

and so

$$
\alpha=\alpha_{0}
$$

almost everywhere on $\Omega$, and hence on $W$. Thus $\alpha \in \Gamma$ and $\alpha_{i k_{j}} \rightarrow \alpha$ weakly. Consequently, $\alpha_{i} \rightarrow \alpha$ weakly since every subsequence contains a subsequence converging weakly to $\alpha$.

We introduce the following notation: If $A$ is a linear subspace of $\Gamma$, then $\bar{A}$ is the smallest closed linear subspace containing $A$ (strong and weak closures 
being the same for linear subspaces by Lemma 4$)$. By $A *$ is meant the linear subspace consisting of all $\alpha$ such that $* \alpha \in A$. If $A$ and $B$ are two linear subspaces, by $A+B$ we mean the linear subspace consisting of all differentials of the form $\alpha+\beta$, with $\alpha \in A$, and $\beta \in B$.

We say that two differentials $\alpha$ and $\beta$ of $\Gamma$ are orthogonal (in symbols $\alpha \perp \beta)$ if $(\alpha, \beta)=0$. We say that a differential $\beta$ is orthogonal to a linear subspace $A$ (written $\beta \perp A$ ) if $\beta \perp \alpha$ for every $\alpha \in A$. Two linear subspaces $A$ and $B$ are orthogonal $(A \perp B)$ if $\alpha \perp B$ for every $\alpha \in A$. Clearly if $A \perp B$, then $\bar{A} \perp \bar{B}$. We write $A \oplus B$ to mean $A+B$ and $A \perp B$. The orthogonal complement $A^{\perp}$ of a linear subspace $A$ is the set of all $\alpha$ such that $\alpha \perp A$. Certainly $A^{\perp}$ is always closed. An important lemma concerning orthogonal complements is the following:

Lemma 9. If $A$ is a closed linear subspace of $\Gamma$, then $\Gamma=A \oplus A^{\perp}$. Consequently, $A^{\perp \perp}=A$.

6. Weak differentials. We use $K$ to denote the class of all piecewise smooth functions with a compact carrier. Then to each locally square integrable $\alpha$ we associate a linear functional

$$
d \alpha[\phi]=-\iint d \phi \alpha
$$

on functions $\phi$ of class $K$. On a bounded Riemann surface we define $d \alpha$ only on those functions of class $K$ which also vanish on the boundary of the Riemann surface. This linear functional is called the weak differential of $\alpha$. If there is a real number $M$ such that

$$
|d \alpha[\phi]| \leqq M
$$

for all $\phi \in K$ with $|\phi| \leqq 1$, then $\alpha$ is called regular. Again an application of Green's theorem gives us the following:

Lemma 10. If $\alpha$ is g.-continuous,

$$
d \alpha[\phi]=\iint \phi d \alpha,
$$

and $\alpha$ is reguilar if and only if

$$
\iint|d \alpha|<\infty
$$

Lemma 11. Let $\phi_{i}$ be a uniformly bounded sequence of functions of class $K$ which converges to zero uniformly on each compact region $\Omega \subset W$. Then if $\alpha$ is regular,

$$
d \alpha\left[\phi_{i}\right] \rightarrow 0
$$


Proof. Without loss of generality we may assume $\left|\phi_{i}\right| \leqq 1$. Let $M$ $=\sup d \alpha[\phi]$ for all $\phi \in K$ with $|\phi|<1$, and given $\epsilon>0$, let $\phi_{0}$ be a function of class $K$ with $\left|\phi_{0}\right|<1$, and $d \alpha\left[\phi_{0}\right]>M-\epsilon$. Let $n$ be chosen so large that $\left|\phi_{i}\right|<\epsilon$ on the carrier of $\phi_{0}$ for all $i \geqq n$. Then

$$
\left|\phi_{i}+(1-\epsilon) \phi_{0}\right| \leqq 1 \text {, }
$$

and hence

$$
\begin{aligned}
M & \geqq d \alpha\left[\phi_{i}+(1-\epsilon) \phi_{0}\right] \\
& =d \alpha\left[\phi_{i}\right]+(1-\epsilon) d \alpha\left[\phi_{0}\right] \\
& \geqq d \alpha\left[\phi_{i}\right]+M-(M+1) \epsilon
\end{aligned}
$$

or

$$
d \alpha\left[\phi_{i}\right] \leqq(M+1) \epsilon
$$

Similarly

$$
d \alpha\left[-\phi_{i}\right] \leqq(M+1) \epsilon
$$

whence

$$
\left|d \alpha\left[\phi_{i}\right]\right| \leqq(M+1) \epsilon
$$

and the lemma is proved.

7. Harmonic differentials and the lemma of Weyl. A first order differential $\omega$ is called harmonic if every point has a neighborhood in which $\omega$ is the differential of a harmonic function. Thus harmonic differentials possess derivatives of all orders. The class of square integrable harmonic differentials will be denoted by $\Gamma_{H}$.

It is readily verified that a necessary and sufficient condition that a first order differential $\omega$ be harmonic is that $\omega \in C^{1}$ and

$$
d \omega=d * \omega=0 .
$$

As a consequence $* \omega$ is harmonic whenever $\omega$ is. Thus in particular $\Gamma_{H}^{*}=\Gamma_{H}$.

If $\phi \in K$, and $\omega$ is harmonic, then Green's theorem easily gives

$$
\iint d \phi * \bar{\omega}=0 \text { and } \iint * d \phi * \bar{\omega}=0 .
$$

As a converse we have the following lemma:

LEMMA 12 (WeYL). Let $\omega$ be a locally square integrable differential such that

$$
\iint d \phi * \bar{\omega}=0 \text { and } \iint * d \phi * \bar{\omega}=0
$$


for every $\phi \in K$. Then $\omega$ is a harmonic differential.

Since the property of being harmonic is purely a local one, it suffices to prove the lemma for a plane region, and the reader is referred to Weyl [38]. It is worth noting that the lemma says that if the weak differentials of $\omega$ and $* \omega$ vanish, then $\omega$ is harmonic.

8. Closed differentials and periods. A first order differential $\alpha$ is called closed if its weak differential vanishes.

Lemma 13. Let $\alpha$ be a closed g.-continuous differential. Then each point has a neighborhood $U$ in which there is a function $f$ defined so that $\alpha=d f$ in $U$.

Proof. Let $U$ be a circle $\left|z-z_{0}\right|<r$. If $J_{1}$ and $J_{2}$ are two piecewise analytic arcs in $U$ with the same beginning and end points, then $J_{1}$ and $-J_{2}$ bound a region $\Omega \subset U$ and

$$
\int_{J_{1}} \alpha-\int_{J_{2}} \alpha=\iint_{\Omega} d \alpha=0
$$

by Green's theorem. Thus if we define

$$
f(p)=\int_{J} \alpha
$$

where $J$ is a piecewise analytic arc beginning at $p_{0}$ and ending at $p$, we have a definition independent of the arc $J$ chosen, and it is easily verified that $f$ is piecewise smooth and that

$$
\alpha=d f \text {. }
$$

We say that a piecewise analytic closed curve $J$ is homologous to zero, or that it bounds, if there is a compact region whose boundary is $J$. We call two closed piecewise analytic curves $J_{1}$ and $J_{2}$ homologous if they form the boundary of a compact region $\Omega$ such that $\Omega$ lies to the right as we traverse $J_{1}$ and to the left as we traverse $J_{2}$ or vice versa. From Green's theorem we have the following lemma:

LEMMA 14. If $J_{1}$ and $J_{2}$ are homologous piecewise analytic closed curves and $\alpha$ a closed g.-continuous differential, then

$$
\int_{J_{1}} \alpha=\int_{J_{2}} \alpha .
$$

Let $h$ be a homology class (class of homologous, piecewise analytic closed curves) and $\alpha$ a closed g.-continuous differential. Then we define the period of $\alpha$ with respect to $h$ as 


$$
P_{h}(\alpha)=\int_{J} \alpha
$$

where $J \in h$. By Lemma 14 the definition is independent of the choice of $J$.

If we wish to extend this concept to closed differentials which are not g.-continuous, we must try to make a definition of $P_{h}(\alpha)$ which does not depend on line integration but rather on area integration. To do this we resort to the following artifice: Suppose $J$ is a piecewise analytic closed curve belonging to the homology class $h$. Then we define a differential $\eta_{h}$ which has a compact carrier and derivatives of all orders as follows: Let $U$ be a compact region containing $J$ which $J$ separates into two parts $U_{1}$ and $U_{2}$. The existence of such a region follows easily from the definition of a piecewise analytic closed curve. In $U_{1}$ we define a function $\phi \in C^{\infty}$ which is identically one in a neighborhood of $J$ and which vanishes identically in a neighborhood of the remainder of the boundary of $U_{1}$. Then $\eta_{k}=-{ }_{*} d \phi$ is a differential, whose dual is closed, of class $C^{\infty}$ whose carrier is contained in $U_{1}$, and hence is compact. If $\alpha$ is a closed g.-continuous differential, we have

$$
\begin{aligned}
\iint \alpha * \eta_{h} & =\iint_{U_{1}} \alpha * \eta_{h} \\
& =\iint_{U_{1}} \alpha d \phi \\
& =\int_{J} \alpha \\
& =P_{h}(\alpha)
\end{aligned}
$$

by Green's theorem, since $\phi=1$ on $J$ and $\phi=0$ on the rest of the boundary of $U_{1}$. Thus we may define

$$
P_{h}(\alpha)=\iint \alpha * \eta_{h}
$$

for all closed differentials $\alpha$ which are locally square integrable, and this corresponds to our earlier definition if $\alpha$ is g.-continuous. It is not apparent whether or not this definition depends on the choice of the functions $\eta_{h}$ if $\alpha$ is an arbitrary locally square integrable closed differential. However, it will be shown in the next section that if $\alpha$ is a closed differential belonging to the space $\Gamma$, then $P_{h}(\alpha)$ is actually independent of the choice of $\eta_{h}$ and depends only on the homology class $h$.

The following lemma is readily proved:

Lemma 15. A closed g.-continuous differential $\alpha$ is the differential of a func- 
tion if and only if

$$
P_{h}(\alpha)=\iint \alpha * \eta_{h} \doteq 0
$$

for each homology class $h$.

9. Orthogonal projection. As in $\$ 7$ we denote by $K$ the class of piecewise smooth functions whose carriers are compact, and we use $\Gamma_{K}$ to denote the subspace of $\Gamma$ consisting of the differentials of functions of class $K$. If $f \in K$, and $\omega \in \Gamma_{H}$, then by Green's theorem we have, since $d * \omega=0$,

$$
(d f, \omega)=\iint_{\Omega} d f * \bar{\omega}=0
$$

where $\Omega$ is a compact region containing the carrier of $f$. Since $* \omega \in \Gamma_{H}$, we also have

$$
(* d f, \omega)=-(d f, * \omega)=0 .
$$

Thus $\Gamma_{H}$ is orthogonal to both $\Gamma_{K}$ and $\Gamma_{K}^{*}$. Now if $f_{1}$ and $f_{2}$ belong to $K$, then

$$
\begin{aligned}
\left(d f_{1}, * d f_{2}\right) & =\iint_{\Omega} d f_{1} * * \overline{d f_{2}} \\
& =-\iint_{\Omega} d f_{1} \overline{d f_{2}}=0
\end{aligned}
$$

by Green's theorem, where $\Omega$ is a compact region containing the carriers of $f_{1}$ and $f_{2}$. Thus $\Gamma_{K}$ and $\Gamma_{K}^{*}$ are orthogonal, and consequently $\Gamma_{H}, \Gamma_{K}$, and $\Gamma_{K}^{*}$ are mutually orthogonal linear subspaces. However, Lemma 12 asserts that if $\omega \perp \Gamma_{K}$ and $\omega \perp \Gamma_{K}^{*}$, then $\omega \in \Gamma_{H}$. Thus

$$
\left(\Gamma_{K}+\Gamma_{K}^{*}\right)^{\perp} \subseteq \Gamma_{H} \subseteq\left(\Gamma_{K}+\Gamma_{K}^{*}\right)^{\perp},
$$

whence $\Gamma_{H}=\left(\Gamma_{K}+\Gamma_{K}^{*}\right)^{\perp}$. Thus in view of Lemma 9 we have established the following proposition:

Proposition 1. The linear subspace $\Gamma_{H}$ is closed, and $\Gamma$ has the orthogonal decomposition

$$
\Gamma=\Gamma_{H} \oplus \bar{\Gamma}_{K} \oplus \bar{\Gamma}_{K}^{*}
$$

When we note that the weak differential of a differential in $\bar{\Gamma}_{K}$ vanishes, this proposition has the following form in terms of differentials.

Proposition 2. If $\alpha \in \Gamma$, then

$$
\alpha=\omega+\alpha_{1}+* \alpha_{2}
$$


where $\omega \in \Gamma_{H}, \alpha_{1}, \alpha_{2} \in \bar{\Gamma}_{K}$. Moreover, the weak differential of $\alpha$ is that of $* \alpha_{2}$ and the weak differential of $* \alpha$ that of $* \alpha_{1}$.

Proposition 3. The space $\Gamma_{c l}$ of closed square integrable differentials has the orthogonal decomposition

$$
\Gamma_{c l}=\Gamma_{H} \oplus \bar{\Gamma}_{K}
$$

Hence $\Gamma_{c l}$ is a closed linear subspace and

$$
\Gamma_{c l}=\left(\Gamma_{K}^{*}\right)^{\perp}=\left(\bar{\Gamma}_{K}^{*}\right)^{\perp} \text {. }
$$

Proof. If $\alpha \in \Gamma_{H} \oplus \bar{\Gamma}_{K}, \alpha$ is certainly closed. On the other hand if $\alpha$ is closed, we decompose $\alpha$ by Proposition 2 into

$$
\alpha=\omega+\alpha_{1}+* \alpha_{2}
$$

whence $* \alpha_{2}=\alpha-\omega-\alpha_{1}$ is closed. But $\alpha_{2} \in \bar{\Gamma}_{K}$, and hence must also be closed. Thus by Lemma 12, $\alpha_{2} \in \Gamma_{H}$. Since $\Gamma_{H}$ and $\bar{\Gamma}_{K}$ are orthogonal, $\alpha_{2}=0$, proving the proposition.

Proposition 4. If $\alpha \in \Gamma$ is closed, then

$$
\alpha=\omega+\alpha_{1}
$$

where $\omega \in \Gamma_{H}$ and $\alpha_{1} \in \bar{\Gamma}_{K}$. The weak differential of $* \alpha$ is that of $* \alpha_{1}$, while the periods of $\omega$ are those of $\alpha$. Consequently, the periods $P_{h}(\alpha)$ depend only on the homology class $h$ and not on the function $\eta_{h}$.

Proof. It is necessary only to show that $\alpha_{1}$ has no periods. But if $\alpha_{1}$ is in $\Gamma_{K}$, then $\alpha_{1}=d f$ with $f$ piecewise smooth, and

$$
\begin{aligned}
P_{h}\left(\alpha_{1}\right) & =\left(\alpha_{1}, \eta_{h}\right) \\
& =\iint \alpha_{1} * \bar{\eta}_{h} \\
& =\int_{J} \alpha_{1} \\
& =\int_{J} d f=0,
\end{aligned}
$$

where $J$ is that piecewise analytic closed curve of class $h$ used in defining $\eta_{h}$. If $\alpha_{1}$ belongs to $\bar{\Gamma}_{K}$ but not to $\Gamma_{K}$, then there is a sequence $\alpha^{\nu} \rightarrow \alpha_{1}, \alpha^{\nu} \in \Gamma_{K}$. But

$$
\left(\alpha^{\nu}, \eta_{h}\right)=0
$$

and hence

$$
\left(\alpha_{1}, \eta_{h}\right)=0
$$


by the continuity of the scalar product.

We define the space $D$ to be the space of all piecewise smooth functions for which $d f \in \Gamma$, and write

$$
D(f, g)=(d f, d g)
$$

and

$$
D(f)=(d f, d f) .
$$

We have the following decomposition theorem for the space $D$ :

Proposition 5. If $f \in D$, then

$$
f=u+f_{0}
$$

where $u$ is a harmonic function and $d f_{0} \in \bar{\Gamma}_{K}$. Moreover, $d u$ and $d f_{0}$ are orthogonal.

Proof. Since $d f \in \Gamma_{c l}$, we have by Proposition 3 that $d f=\omega+\alpha_{1}$ where $\omega \in \Gamma_{H}$, and $\alpha_{1} \in \bar{\Gamma}_{K}$. Furthermore, $\omega$ has no period since $d f$ has none. Thus by Lemma 15 and the fact that $\omega \in C^{\infty}, \omega=d u$, where $u$ is a harmonic function. If we set $f_{0}=f-u$, then $d f_{0}=\alpha_{1} \in \bar{\Gamma}_{K}$ and the theorem is proved.

10. The Dirichlet principle. One of the more powerful tools at the disposal of the analyst is the Dirichlet principle. Formulated first by Lord Kelvin in 1847 , it was used extensively by both Dirichlet and Riemann. We shall use it in the following form:

THEOREM 1. Let $\Omega$ be a compact region with boundary $R$ on a Riemann surface, and let $g$ be a piecewise smooth function on $\bar{\Omega}$. Then there is a unique piecewise smooth function $u$ on $\bar{\Omega}$ which is harmonic in $\Omega$ and such that $g-u$ vanishes identically on $R$. Moreover, $D_{\Omega}(u) \leqq D_{\Omega}(g)$.

Proof. Considering $\Omega$ as a Riemann surface in its own right, we apply Proposition 5 to the function $g$ to get

$$
g=v+g_{0}
$$

where $v$ is harmonic and $d g_{0} \in \Gamma_{K}(\Omega)$, i.e., there is a sequence $\phi_{i}$ of piecewise smooth functions whose carriers are contained in $\Omega$ such that $D\left(g_{0}-\phi_{i}\right) \rightarrow 0$. Since $d v$ and $d g_{0}$ are orthogonal in $\Gamma(\Omega), D(g)=D(v)+D\left(g_{0}\right)$. Hence to prove the theorem it suffices to show that $g-v$ approaches a constant as we approach $R$. The proof of this fact is somewhat laborious, and we refer the reader to $[10]$.

This theorem shows the utility of piecewise smooth functions by the fact that if we have a piecewise smooth function $f$ defined on the Riemann surface $W$ and a compact region $\Omega \subset W$, then we can define a new function $f_{1}$ which is again piecewise smooth and which is harmonic in $\Omega$ and identically equal to $f$ outside $\Omega$. Moreover $D\left(f_{1}\right) \leqq D(f)$.

By doubling $\Omega$ with respect to $R_{1}$, we establish the following generalization of Theorem 1: 
THEOREM 1. Let $\Omega$ be a compact region whose boundary consists of the two parts $R_{1}$ and $R_{2}$, and let $g$ be a piecewise smooth function on $\bar{\Omega}$. Then there is a piecewise smooth function $u$ harmonic in $\Omega$ which has a vanishing normal derivative on $R_{1}$, while $g-u$ vanishes on $R_{2}$. Moreover $D(u) \leqq D(g)$.

If $\Omega$ is a compact region with boundary $R$, we call $g(p, q)$ its Green's function if it is harmonic (as a function of $p$ ) in $\Omega-q$, approaches zero as $p \rightarrow R$, and has a logarithmic pole at $q$, i.e., for a uniformizer $z$ at $q$ we have $g(p, q)+\log |z(p)-z(q)|$ a harmonic function near $q$. We have the following well known proposition:

PROPOSITION 6. Given a compact region $\Omega$ and a point $q \in \Omega$, there exists a Green's function $g(p, q)$. Moreover, we have

$$
g(p, q)=g(q, p) .
$$

11. The capacity of a ring domain. If $\Omega$ is a compact region whose boundary consists of two disjoint nonempty compact sets $R_{1}$ and $R_{2}$, we call the triad consisting of $\Omega, R_{1}$, and $R_{2}$ a ring domain. For example, a region in the plane bounded by a finite number of analytic curves becomes a ring domain when the boundary curves are divided into two nonempty classes. The reader should have little difficulty in constructing a piecewise smooth function $g$ on $\bar{\Omega}$ which has the value one on $R_{1}$ and vanishes on $R_{2}$. As a consequence of the Dirichlet principle (Theorem 1 ) there is a harmonic function $u$ with a finite Dirichlet integral which is one on $R_{1}$ and zero on $R_{2}$. This function is called the harmonic measure of $R_{1}$ with respect to $\Omega$ and written $u=u\left(p, R_{1}, \Omega\right)$.

We call

$$
C=D(u)=\iint_{\Omega} d u * d u
$$

the capacity of the ring domain. Among the homology classes of piecewise analytic closed curves in $\Omega$, let $h_{0}$ denote that one to which $R_{1}$ belongs when traversed in such a direction that $\Omega$ lies to the left. Then it is easily verified that $P_{h_{0}}(u)=C$.

Proposition 7. If $\alpha \in \Gamma(\Omega)$, and if $\left({ }^{2}\right)$

$$
\int_{J} \alpha \geqq 1
$$

for every piecewise analytic curve $J$ beginning on $R_{2}$ and ending on $R_{1}$, then

$$
\iint_{\Omega} \alpha * \alpha \geqq \iint_{\Omega} d u * d u=C
$$

(2) We thus implicitly impose upon $\alpha$ the condition that for all piecewise analytic arcs beginning on $R_{2}$ and ending on $R_{1}$ the integral must either exist or diverge to $+\infty$. 
with equality only if $\alpha=d u$ almost everywhere.

Proposition 7'. If $\beta \in \Gamma(\Omega)$, and if

$$
\int_{J} \beta \geqq 1
$$

for all $J \in h_{0}$, then

$$
\iint \beta * \beta \geqq \iint \frac{d u}{C} * \frac{d u}{C}=\frac{1}{C}
$$

with equality only if $\beta=* d u / C$ almost everywhere.

These propositions give upper and lower bounds for the capacity of a ring domain and are known in electricity (in a somewhat less general form) as Dirichlet's and Thomson's principles respectively, although both are due to W. Thomson, Lord Kelvin. Since the proofs of the two propositions are nearly identical, we prove the former and leave the latter to the reader. The proof given below is modeled after a proof given by Ahlfors and Beurling in the theory of extremal distances. The central idea of the proof, however, stems from the length-area principle of Grötzsch.

First I maintain that $u$ can have only a finite number of critical points, i.e., points where $d u=0$. For if there were an infinite number, they must have a limit point, $\bar{\Omega}$ being compact. If this limit point were an interior point of $\Omega$, we would have a neighborhood of it in which $u$ is a harmonic function of the uniformizer variable which has a limit point of critical points as a point of harmonicity, when $u$ would be identically constant, which is impossible. On the boundary of $\Omega$, we note that $u$ is a harmonic function of the boundary uniformizer by the Schwarz reflection principle, and the same argument applies.

Let $v$ be the conjugate function of $u$, i.e., the function such that $d v=* d u$. Then $v$ is possibly a many-valued function, but the curves where $v=$ constant are well determined. Let $\Omega^{\prime}$ be a subregion of $\Omega$ bounded by curves $J_{0}$ on which $v$ is constant and such that $\Omega^{\prime}$ contains no critical points of $u$. Clearly

$$
\iint_{\Omega^{\prime}} d u * d u \rightarrow C,
$$

and

$$
\iint_{\Omega^{\prime}} \alpha * \alpha \rightarrow \iint_{\Omega} \alpha * \alpha
$$

as $\Omega^{\prime} \rightarrow \Omega$. But in $\Omega^{\prime}$ we may, in each sufficiently small neighborhood, use $u+i v$ as a uniformizer ( $u$ and $v$ are seen to be real from their definition). Thus if $\alpha=a d u+b d v$, we have 


$$
1 \leqq \int_{0}^{1} a d u,
$$

since the curves $J$ where $v=$ constant are analytic curves beginning on $R_{2}$ and ending on $R_{1}$. By the Schwarz inequality

$$
1 \leqq \int_{0}^{1}|a|^{2} d u
$$

and if $J^{\prime}$ denotes that portion of a curve $u=$ constant which lies in $\Omega^{\prime}$,

$$
\begin{aligned}
\iint d u d v & =\iint_{J^{\prime}} d v \\
& \leqq \iint_{\Omega^{\prime}}|a|^{2} d u d v \\
& \leqq \iint_{\Omega^{\prime}}\left(|a|^{2}+|b|^{2}\right) d u d v \\
& =\iint_{\Omega^{\prime}} \alpha * \alpha
\end{aligned}
$$

which, if we let $\Omega^{\prime}$ tend to $\Omega$, proves the theorem.

Chapter III. The space $B D$ on an open Riemann surface

12. The space $B$. In $\$ 9$ we obtained some results concerning the class $D$ of all piecewise smooth functions whose differentials belong to $\Gamma$. However the space $D$ with the topology defined by

$$
f_{i} \rightarrow f
$$

whenever

$$
d f_{i} \rightarrow d f
$$

in $\Gamma$

has two disadvantages: A sequence in which $f_{i}=f$ converges to $f+c$ as well as to $f$, and this topology gives no information concerning the upper bound of the absolute value of a function, a quantity most naturally associated with functions on an arbitrary topological space. This leads us to consider the space $B=B(W)$ consisting of all piecewise smooth functions on the Riemann surface $W$ which are bounded there. When it comes to defining a topology in $B$, the first idea to come to mind is probably to make $B$ a metric space by defining a norm

$$
\|f\|=\sup |f|
$$

but this has the disadvantage that we cannot approach all functions in $B$ 
by means of sequences of functions which vanish or are constant outside a compact region, these being the functions on $W$ about which we have the most complete information. In order to overcome these disadvantages we shall make $B$ into a convergence space by defining

$$
f_{i} \rightarrow f
$$

in $B$

whenever $\left|f_{i}\right|$ is uniformly bounded and $f_{i} \rightarrow f$ uniformly on every compact region.

The reader should be warned that if we define the closure of a set of elements in $B$ as the the adjunction of the limit elements, then the closure of a closed set need not be closed, as simple examples will show. Actually it is customary to define the closure of a set as the smallest closed set containing the given set, a set being closed if it contains all of its limit elements. In this case, however, it must be remembered that not all frontier elements of a set are accessible by sequences of elements belonging to the set. That is, equivalently, neither countability axiom is satisfied.

In $\S 6$ we introduced the weak differential of a locally square integrable differential as a linear functional on the space $K$ defined by

$$
d \alpha[\phi]=-\iint d \phi \alpha
$$

and saw (Lemma 11) that if $\phi_{i} \in K$ and

$$
\phi_{i} \rightarrow 0
$$

in $B$,

then

$$
d \alpha\left[\phi_{i}\right] \rightarrow 0
$$

for each regular $\alpha$.

LEMмa 16. The weak differential of a locally square integrable differential $\alpha$ admits of a unique extension to the space $B$ which is continuous in the sense that

$$
d \alpha\left[f_{i}\right] \rightarrow d \alpha[f]
$$

whenever

$$
f_{i} \rightarrow f \quad \text { in } B .
$$

Proof. Let $f \in B$. Then there is easily constructed a sequence $\phi_{i} \in K$ such that

$$
\phi_{i} \rightarrow f \quad \text { in } B .
$$

Now

$$
\phi_{i}-\phi_{j} \rightarrow 0
$$


as $i, j \rightarrow \infty$. By Lemma 11

$$
d \alpha\left[\phi_{i}-\phi_{j}\right] \rightarrow 0,
$$

and hence $d \alpha\left[\phi_{i}\right]$ converges to a number which we take as the definition of $d \alpha[f]$. This definition is independent of the sequence chosen since the difference of any two such sequences must tend to zero in $B$ and hence lead to the same definition of $d \alpha[f]$.

Now it is clear from the definition that

$$
\max _{|f| \leqq 1}|d \alpha[f]|=\max _{|\phi| \leqq 1}|d \alpha[\phi]| \quad \phi \in K .
$$

Thus the same proof as was used for Lemma 11 shows that if

$$
f_{i} \rightarrow 0 \quad \text { in } B,
$$

then

$$
d \alpha\left[f_{i}\right] \rightarrow 0,
$$

from which the required continuity of $d \alpha$ follows, proving the lemma.

We shall say that

$$
f_{i} \rightarrow f \quad \text { weakly in } B
$$

if for every regular $\alpha$

$$
d \alpha\left[f_{i}\right] \rightarrow d \alpha[f] .
$$

Clearly convergence in $B$ implies weak convergence in $B$. It should be noted, however, that our definition of weak convergence differs from the standard definition of weak convergence in a linear vector space which requires

$$
L\left[f_{i}\right] \rightarrow L[f]
$$

for all continuous linear functionals $L$. However, the definition which we have given is the one that proves to be most useful for our purposes.

We note in passing that both weak topologies are actually the same. Indeed, it can be shown, although with considerable effort, that the convergence of $f_{i}$ to $f$ in either weak topology is equivalent to having $\left|f_{i}\right|$ uniformly bounded and $f_{i}$ converging to $f$ everywhere. However, the use of the definition of weak convergence which we have given absolves us of the need of proving this fact here.

13. The space $B D$. We shall obtain more fruitful results, however, when we consider the space $B D$ consisting of all bounded, piecewise continuously differentiable functions $f$ for which $d f$ belongs to $\Gamma$, i.e., consisting of these functions which belong to both $B$ and $D$. We introduce a topology in the space $B D$ by defining 
whenever

$$
f_{i} \rightarrow f
$$

and

$$
d f_{i} \rightarrow d f
$$

in $\Gamma$.

We shall also introduce a weak topology by saying that

$$
f_{i} \rightarrow f
$$

weakly in $B D$

whenever

$$
f_{i} \rightarrow f
$$

weakly in $B$

and

$$
d f_{i} \rightarrow d f
$$

weakly in $\Gamma$.

As in $\S 6$, we denote by $K$ the subclass of $B D$ consisting of those functions with a compact carrier. We say that a function $f$ of class $B D$ is of class $\bar{K}$ if there is a sequence of functions $\phi_{i}$ in $K$ such that

$$
\phi_{i} \rightarrow f
$$

As was mentioned before, there is a priori no reason to suppose that $\bar{K}$ is closed. In the next section, however, it will be shown that $\bar{K}$ is actually closed in the weak topology of $B D$ and hence also in the strong topology. Clearly $\Gamma_{\bar{K}} \subset \bar{\Gamma}_{K}$. The following proposition shows that functions of the class $\bar{K}$ behave in some respects as though they "had zero boundary values":

PRoposition 8. If $\alpha \in \Gamma$ is a regular differential, and if $f \in \bar{K}$, then

$$
d \alpha[f]=-\iint d f \alpha .
$$

Proof. There is a sequence $\phi_{i} \in K$ such that $\phi_{i} \rightarrow f$ in $B D$. Now for $\phi_{i} \in K$, we have by definition

$$
d \alpha\left[\phi_{i}\right]=-\iint d \phi_{i} \alpha
$$

But

$$
d \alpha\left[\phi_{i}\right] \rightarrow d \alpha[f]
$$

since $\phi_{i} \rightarrow f$ in $B$, and

$$
\iint d \phi_{i} \alpha \rightarrow \iint d f \alpha
$$

since $d \phi_{i} \rightarrow d f$ in $\Gamma$. 
As a direct consequence of Lemmas 5, 7, and 8, we have the following condition for convergence:

LEMMA 17. Let $\left\{f_{i}\right\}$ be a uniformly bounded sequence of functions of the space $B D$ which converge uniformly together with their derivatives on every compact region to a function $f$. If $D\left(f_{i}\right)$ is uniformly bounded, then $f \in B D$, and

$$
f_{i} \rightarrow f
$$

weakly in $B D$.

Moreover,

$$
D(f) \leqq \lim \inf D\left(f_{i}\right)
$$

If, furthermore,

$$
D\left(f_{i}-f_{j}\right) \rightarrow 0
$$

as $i, j \rightarrow \infty$, then

$$
f_{i} \rightarrow f
$$

strongly in $B D$.

If we use $H B D$ to denote the space of harmonic functions which belong to the space $B D$, we have the following decomposition theorem:

THEOREM 2. If $f \in B D$, then

$$
f=f_{K}+u,
$$

where $f_{K} \in \bar{K}$ and $u \in H B D$. Moreover

$$
\|u\| \leqq\|f\| .
$$

Proof. Choose an exhaustion $\left\{\Omega_{i}\right\}$ of $W$, and set $f=\phi_{i}+u_{i}$, where $u_{i}$ is that piecewise smooth function which is identically equal to $f$ outside $\Omega_{i}$ and harmonic inside $\Omega_{i}$. The existence of $u_{i}$ is guaranteed by Theorem 1 . Since on every compact region $u_{i}$ is eventually a uniformly bounded (by $\|f\|$ ) sequence of harmonic functions, there is by Lemmas 1 and 2 a subsequence (which we shall again call $u_{i}$ ) which converges in $B$ to a harmonic function $u$, and for which $d u_{i}$ converges to $d u$ uniformly on every compact set.

By the Dirichlet principle $D\left(u_{j}\right) \leqq D\left(u_{i}\right)$ for $i<j$, whence $D\left(u_{i}\right)$ converges. Since $u_{i}-u_{j}$ vanishes outside $\Omega_{j}$ while $u_{j}$ is harmonic inside $\Omega_{j}$, we have by Green's theorem that

$$
0=D\left(u_{j}, u_{i}-u_{j}\right)=D\left(u_{i}, u_{j}\right)-D\left(u_{j}\right) .
$$

Hence

$$
\begin{aligned}
D\left(u_{i}-u_{j}\right) & =D\left(u_{i}\right)-2 D\left(u_{i}, u_{j}\right)+D\left(u_{j}\right) \\
& =D\left(u_{i}\right)-D\left(u_{j}\right) .
\end{aligned}
$$

Thus $d u_{i}$ converges in $\Gamma$, and by Lemma 17 


$$
\phi_{i} \rightarrow f_{K}=f-u
$$

Consequently, $f_{K} \in \bar{K}$, since $\phi_{i} \in K$.

CoRollary 1. If $f \in B D$ and $d f \in \bar{\Gamma}_{K}$, then there is a constant $c$ such that $f-c \in \bar{K}$.

Proof. In the decomposition $f=f_{K}+u$ we must have $d u=0$, since $\Gamma_{H}$ and $\Gamma_{K}$ are both orthogonal while $d f$ and $d f_{K}$ both belong to $\bar{\Gamma}_{K}$.

Corollary 2. We have

$$
\Gamma_{\bar{K}}=\bar{\Gamma}_{K} .
$$

14. Harmonic measures and the type of a Riemann surface. We shall say that an open Riemann surface $W$ is of parabolic type if $1 \in \bar{K}$. Otherwise we say that $W$ is of hyperbolic type. In the next section it will be shown that this definition is equivalent to the usual one in terms of the existence of Green's functions. It should be noted that if $1 \in \bar{K}$, then by Prosposition 8

$$
d \alpha[1]=-\iint d 1 \alpha=0
$$

for every regular differential $\alpha \in \Gamma$. In order to establish the converse of this we first establish some theorems concerning harmonic measure.

Let $C$ be a set consisting of a finite number of piecewise analytic closed curves, and let $W_{1}$ be an open subset of $W$ which is closed in $W-C$, i.e., which is bounded by a part of $C$. Let $\left\{\Omega_{i}\right\}$ be a sequence of compact regions such that $\bar{\Omega}_{i} \subset \Omega_{i+1}, C \cap \bar{W}_{1} \subset \bar{\Omega}_{1}$, and $U \Omega_{i}=W_{1}$. The existence of such a sequence is a consequence of the compactness of $C$. In each $\Omega_{i}$ we construct that function $u_{i}$ which is piecewise smooth on $W_{1}$, harmonic in $\Omega_{i}$, has the boundary value one on $C$, and vanishes identically in $W_{1}-\Omega_{i}$. The existence of $u_{i}$ is again a simple consequence of the compactness of $C$. By the maximum principle

$$
0 \leqq u_{i} \leqq u_{j} \leqq 1
$$

in $\Omega_{i}$ provided that $i<j$. By Harnack's theorem (Lemmas 1 and 2 ) the functions $u_{i}$ converge in $B$ to a harmonic function $u$, while $d u_{i}$ converges to $d u$ uniformly on every compact set of $W_{1}$. By the Dirichlet principle (Theorem 1) we have

$$
D\left(u_{i}\right) \geqq D\left(u_{j}\right)
$$

for $i<j$. Hence $D\left(u_{i}\right)$ converges. But if $i<j$, we have by Green's theorem

$$
\begin{aligned}
D\left(u_{i}-u_{j}\right) & =D\left(u_{i}\right)-D\left(u_{j}\right)+2 D\left(u_{j}, u_{j}-u_{i}\right) \\
& =D\left(u_{i}\right)-D\left(u_{j}\right)
\end{aligned}
$$


whence $d u_{i}$ converges in $\Gamma\left(W_{1}\right)$.

The function $u$ which we have constructed is called the harmonic measure of $C$ with respect to $W_{1}$ and is denoted by the expression $u\left(p, C, W_{1}\right)$. That this harmonic measure does not depend on the particular exhaustion of $W_{1}$ which is chosen is a consequence of the fact that the harmonic measure is characterized by being the smallest non-negative piecewise smooth function on $\bar{W}_{1}$ which is harmonic in $W_{1}$ and identically equal to one on $C$.

An important special case is that in which $C$ is the boundary of a compact region $\Omega$, and $W_{1}=W-\bar{\Omega}$. In this case $u$ is simply called the harmonic measure of $\Omega$, and written $u(p, \Omega)$. In the remainder of this chapter we restrict ourselves to harmonic measures of compact regions. If we define $u$ and its approximating functions $u_{i}$ to be identically one on the compact region $\Omega$, then $u$ and $u_{i}$ are piecewise smooth on $W$ and

$$
u_{i} \rightarrow u
$$

in $B D$.

But the $u_{i}$ are functions of the class $K$. Hence we have proved the following proposition:

PROPOSITION 9. The harmonic measure $u=u(p, \Omega)$ of a compact region belongs to the class $\bar{K}$.

THEOREM 3. If some compact region $\Omega \subset W$ has a constant harmonic measure, then $W$ is parabolic. If $W$ is of parabolic type, then the harmonic measure of each compact region $\Omega \subset W$ is identically equal to one.

Proof. If $u(p, \Omega)$ is constant, it must be identically one since $u=1$ on $C$. Thus $1=u(p, \Omega)$ belongs to $\bar{K}$ by Proposition 9 .

Suppose on the other hand that $1 \in \bar{K}$. Then there is a sequence $\phi_{i} \in K$ such that

$$
\phi_{i} \rightarrow 1
$$

in $B D$.

Let $\Omega_{i}$ be a compact region containing a given compact region $\Omega$ and the carriers of all $\phi_{j}$ with $j \leqq i$. Since $\phi_{i} \rightarrow 1$ uniformly on $\Omega$, we may insure that $\phi_{i}>1-\epsilon$ on $\Omega$ simply by omitting a finite number of terms from the sequence $\left\{\phi_{i}\right\}$. Now $\Omega_{i}-\Omega$ becomes a ring domain if we take $R_{1}$ to be the boundary of $\Omega$ and $R_{2}$ to be the boundary of $\Omega_{i}$. On $R_{1}$ we have $\phi_{i} \geqq 1-\epsilon$, while $\phi_{i}$ vanishes on $R_{\mathbf{2}}$. Hence by Proposition 7

$$
D\left(\phi_{i}\right) \geqq(1-\epsilon)^{2} D\left(u_{i}\right),
$$

where we have set $u_{i}$ equal to the harmonic measure of $R_{1}$ with respect to $\Omega_{i}-\Omega$. Hence $D\left(u_{i}\right) \rightarrow 0$, since $D\left(\phi_{i}\right) \rightarrow 0$. But

$$
u_{i} \rightarrow u(p, \Gamma)
$$

in $B D$,

whence $D(u)=0$. Therefore $u$ is identically constant, and thus $u=1$ iden- 
tically, proving the theorem.

THEOREM 4. A necessary and sufficient condition that a Riemann surface $W$ be hyperbolic is the existence of a regular differential $\alpha \in \Gamma$ such that

$$
d \alpha[1] \neq 0 .
$$

Proof. As we noted before $d a[1]=0$ for every regular differential $\alpha \in \Gamma$ on a parabolic Riemann surface. Thus it remains only to prove that on a hyperbolic Riemann surface there is a regular differential $\alpha \in \Gamma$ such that $d \alpha[1] \neq 0$.

Let $U$ be a neighborhood $|z|<r$ in which the uniformizer $z$ is valid, and let $\Omega$ be the compact region $|z|<r^{\prime}<r$. If we set $u=u(p, \Omega)$, we know by Theorem 3 that $u$ is not constant. Moreover, $u$ is a harmonic function in the annulus $r^{\prime}<|z|<r$ and is identically one on $|z|=r^{\prime}$. Thus by the Schwarz reflection principle $u$ has a continuous normal derivative on $|z|=r^{\prime}$, i.e., $* d u$ is g.-continuous in $W-\Omega$. Therefore, we have by Green's theorem

$$
\begin{aligned}
d * d u[\phi] & =-\iint_{W} d \phi * d u=-\iint_{W-\Omega} d \phi * d u \\
& =-\int_{|z|=r^{\prime}} \phi * d u
\end{aligned}
$$

for a function $\phi \in K$, and so $* d u$ is regular. But

$$
d * d u[1]=-\int_{|z|=1} * d u=-D(u)<0 .
$$

COROLlary. If $W$ is parabolic, then there are no nonconstant harmonic functions in the class $B D$.

Proof. If $u$ were such a function, then $u * d u$ is a regular differential belonging to $\Gamma$, and

$$
d(u * d u)[1]=\iint d u * d u \neq 0 .
$$

We are now in a position to prove the following proposition.

Proposition 10. The space $\bar{K}$ is a weakly closed linear subspace of $B D$; i.e., if $f \in B D$ and there is a sequence $f_{i} \in \bar{K}$ such that

$$
f_{i} \rightarrow f
$$

weakly in $B D$,

then $f \in \bar{K}$.

Proof. Since $\bar{\Gamma}_{K}$ is a strongly closed linear subspace of $\Gamma$, it is also weakly closed by Lemma 4 . Thus $d f \in \bar{\Gamma}_{K}$. By the first corollary of Theorem 2 we may 
write $f=f_{K}+c$, whence $f_{K} \in \bar{K}$. If $W$ is parabolic, then $c \in \bar{K}$, and so $f \in \bar{K}$.

If $W$ is hyperbolic, let $\alpha$ be a regular differential in $\Gamma$ with $d \alpha[1] \neq 0$. Now

$$
\begin{aligned}
d \alpha[f] & =\lim d \alpha\left[f_{i}\right] \\
& =-\lim \iint d f_{i} \alpha \\
& =-\iint d f \alpha
\end{aligned}
$$

by weak convergence in $B D$ and Proposition 8 . Also by Proposition 8

$$
d \alpha\left[f_{K}\right]=-\iint d f_{K} \alpha .
$$

Hence, since $c=f-f_{K}$,

$$
d \alpha[c]=-\iint d c \alpha=0 .
$$

Consequently, $c=0$ since $d \alpha[c]=c d \alpha[1]$. Thus $f=f_{K} \in \bar{K}$.

15. Green's functions on an open Riemann surface. Let $\left\{\Omega_{i}\right\}$ be an exhaustion of a Riemann surface $W$ such that $\Omega_{1}$ contains the point $q$. Let $G_{i}(p, q)$ be the Green's function of $\Omega_{i}$ with a pole at $q$. By the maximum principle $G_{i}(p, q)$ is a monotone increasing sequence of harmonic functions, and so by Harnack's theorem either $G_{i}(p, q)$ converges together with its derivatives to a harmonic function $G(p, q)$, the convergence being uniform on each compact set not containing $q$, or else $G_{i}(p, q)$ diverges to $+\infty$ uniformly on every compact set. In the first case we call $G(p, q)$ the Green's function for $W$ with a pole at $q$. The Green's function clearly has the property of being the smallest non-negative function on $W$, which is harmonic except at $q$ and which has a logarithmic singularity there. Thus the function $G(p, q)$ is independent of the exhaustion used in its definition.

Proposition 11. If $G(p, q)$ exists for some $q$, then $W$ is of hyperbolic type, while if $W$ is of hyperbolic type, then $G(p, q)$ exists for every $q$.

Proof. If $G(p, q)$ does not exist for some $q$, let

$$
\gamma_{i}=\min \left[1, G_{i}(p, q)\right] .
$$

Since $G_{i}(p, q)$ diverges to $+\infty$ uniformly on every compact region, $\gamma_{i}$ must be identically one on each compact region provided that $i$ is large enough. By Green's theorem

$$
D\left(\gamma_{i}\right)=\int_{G_{i}=1} * d G_{i}=2 \pi .
$$


Thus by Lemma $8, d \gamma_{i} \rightarrow 0$ weakly in $\Gamma$, and by Proposition 10 it follows that $1 \in \bar{K}$, since 1 is the limit of $\gamma_{i}$ in $B$. Thus $W$ is parabolic.

On the other hand, if $G(p, q)$ exists for some $q$, then $G_{i}(p, q)$ converges together with its derivatives to $G(p, q)$ uniformly on each compact set not containing $q$. From Lemma 8 it follows that $G(p, q)$ has a finite Dirichlet integral over any region not containing $q$. Thus if we let $g$ be a twice continuously differentiable function which is identically equal to $G(p, q)$ outside a compact region $\Omega$, then $* d g$ is a regular differential belonging to $\Gamma$. By Green's theorem

$$
d * d g[1]=\int_{R} * d g=2 \pi,
$$

where $R$ is the boundary of $\Gamma$. Thus by Theorem 4 we have $W$ hyperbolic.

This proposition shows that our definition of the type of a Riemann surface is equivalent to the usual one, but has the advantage of being independent from the start of such questions as the dependence of the Green's function on the parameter.

PROPOSITION 12. If $c$ is so large that $G(p, q)>c$ only in a compact region containing $q$, and if $\gamma=\min [c, G]$, then $\gamma \in \bar{K}$.

Proof. If $\gamma_{i}=\min \left[c, G_{i}\right]$, it is easily verified that

$$
\gamma_{i} \rightarrow \gamma
$$

weakly in $B D$.

Since $\gamma_{i} \in K$, we have from Proposition 10 that $\gamma \in \bar{K}$.

Proposition 13. Let $\alpha \in \Gamma$ have the orthogonal decomposition

$$
\alpha=\omega+\alpha_{1}+* \alpha_{2}
$$

of Proposition 1, and suppose that $\alpha_{1}=d f$ where $f \in \bar{K}$. Then

$$
\iint \alpha * d G(p, q)
$$

exists and is equal to $2 \pi f(q)$.

Proof. Clearly

$$
\iint * \alpha_{2} * d G=0
$$

since any approximation to $G$ has a closed differential and $* \alpha_{2}$ is orthogonal to all closed differentials. If we take a small circular neighborhood of $q$ and take $c$ to be larger than the maximum of $G$ on the boundary of this neighborhood, it is readily verified that the region $\Omega$ where $G>c$ is simply connected. By the Riemann mapping theorem we may choose a uniformizer $z$ 
so that $\Omega$ is the set $|z|<1$, and $z(q)=0$. Thus

$$
\begin{aligned}
\iint \alpha * d G & =\iint(\omega+d f) * d G \\
& =\iint(\omega+d f) * d \gamma+\iint(\omega+d f) * d(G-\gamma) .
\end{aligned}
$$

Now

$$
\iint \omega * d \gamma=0
$$

since $\gamma \in K$, and therefore $d \gamma$ is orthogonal to $\omega$. By Proposition 8 and Green's theorem

$$
\iint d f * d \gamma=-d * d \gamma[f]=\int_{R} f * d G
$$

where $R$ is the boundary of $\Omega$. Now $G-\gamma$ vanishes on $R$ and is harmonic in $\Omega$ except for a logarithmic pole at $z=0$. Hence $G-\gamma=-\log |z|$, and we have

$$
\begin{aligned}
-\iint_{\Omega}(\omega+d f) * d \log |z| & =-\iint_{\Omega} d(u+f) * d \log |z| \\
& =2 \pi[u(0)+f(0)]-\int_{R}(u+f) d \phi \\
& =2 \pi f(q)-\int_{R} f d \phi \\
& =2 \pi f(q)-\int_{R} f * d G,
\end{aligned}
$$

where $u$ is a harmonic function such that $\omega=d u$ in $\Omega$, and the proposition follows by adding (1) and (2).

Proposition 14. We have

$$
G(p, q)=G(q, p) .
$$

Proof. Consider the symmetric function

$$
I(p, q)=\iint d_{r} G(r, p) * d_{r} G(r, q)=\iint d_{r} G(r, q) * d G_{r}(r, p) .
$$

Both integrals exist for $p \neq q$ since the differential of a Green's function is dominated by $|d z| /|z|$ at a pole. Hence if we set $\gamma(c)=\min [c, G(r, p)]$ and $\gamma^{\prime}\left(c^{\prime}\right)=\min \left[c^{\prime}, G(r, q)\right]$, 


$$
I(p, q)=\lim _{c, c^{\prime} \rightarrow \infty} \iint d \gamma * d \gamma^{\prime} .
$$

Letting $c^{\prime} \rightarrow \infty$, we have from Proposition 13 that

$$
I(p, q)=\lim _{c \rightarrow \infty} \gamma(c)=G(p, q),
$$

and the symmetry of $G$ follows from that of $I$.

16. The classes $H B D$ and $H D$.

Proposition 15. A necessary and sufficient condition for the existence of a nonconstant function of class $H B D$ is the existence of a regular differential $\alpha \in \Gamma$ and a function $f \in B D$ such that

$$
d \alpha[1]=0
$$

and

$$
d \alpha[f] \neq-\iint d f \alpha .
$$

Proof. By Theorem 2 we have $f=f_{K}+u$, with $u \in H B D$.

$$
\begin{aligned}
d \alpha[u] & =d \alpha\left[f-f_{K}\right] \\
& =d \alpha[f]-d \alpha\left[f_{K}\right] \\
& =d \alpha[f]+\iint d f_{K} \alpha
\end{aligned}
$$

by Proposition 8 . Hence

$$
d \alpha[u]+\iint d u \alpha=d \alpha[f]+\iint d f \alpha \neq 0 .
$$

Therefore $u$ cannot be constant for

$$
d \alpha[c]=0=-\iint d c \alpha .
$$

On the other hand, if $u$ is a nonconstant $H B D$ function, then $\alpha=* d u$ and $f=u$ satisfy the requirements of the theorem.

We denote by $H D$ those functions $f$ for which $d f \in \Gamma_{H}$. The following proposition shows that the space $H D$ is essentially the same as the space $H B D$ :

Proposition 16. If $u \in H D$, then there exists a sequence $u_{i} \in H B D$ such that $d u_{i} \rightarrow d u$ in $\Gamma$.

Proof. Let $A$ be the space of all differentials of functions of class $H B D$ 
and $A^{\prime}$ the space of all differentials of functions of class $H D$. If $\bar{A}^{\prime}$ were different from $\bar{A}$, then there would be a harmonic function $u$ such that $d u \in A^{\prime}$ and $d u \perp A$. But if we set, for $c>c_{1}$,

$$
f_{1}=\max \left[u, c_{1}\right] \quad \text { where } c_{1}<\sup u
$$

and

$$
f=\min \left[f_{1}, c\right] \quad \text { where } c>\inf f_{1},
$$

then $f \in B D$, and $f$ is not constant. Hence $f=f_{K}+u_{1}$, where $u_{1} \in H B D$ and $f_{K} \in \bar{K}$. Now

$$
\iint d u_{1} * d \bar{u}=0
$$

by orthogonality, and

$$
\iint d f_{K} * d \bar{u}=d * d \bar{u}\left[f_{K}\right]=0
$$

by Proposition 8 . Therefore

$$
\iint d f * d \bar{f}=\iint d f * d \bar{u}=0,
$$

a contradiction, since $f$ was not constant. Hence the proposition.

17. Summary. Since Proposition 11 shows that a Riemann surface is hyperbolic if and only if it possesses a Green's function, we shall use $N_{G}$ to denote the class of parabolic Riemann surfaces. Similarly, we use $N_{H D}$ and $N_{H B D}$ to denote the classes of Riemann surfaces whose only $H D$ and $H B D$ functions are constants. By Proposition 16 and the corollary to Theorem 4 we have

$$
N_{G} \subset N_{H D}=N_{H B D} .
$$

We shall return to the classification of Riemann surfaces again in $\$ 23$.

Theorem 2 tells us that

$$
B D=\bar{K} \oplus H B D,
$$

while on parabolic surfaces $B D=\bar{K}$, by the corollary to Theorem 4 . Hence we may say that the surfaces of class $N_{G}$ are characterized by having $H B D$ empty, while the surfaces of class $N_{H B D}$ may be characterized by having $H B D$ consist of at most constants.

There is a rather striking analogy between the behavior of $B D$ functions on an arbitrary Riemann surface and the classical case of $B D$ functions on a plane region bounded by $n$ continua. In this case Theorem 2 asserts nothing more than the solvability of the Dirichlet problem, while in passing to an 
arbitrary Riemann surface we define a function to have "zero boundary values" if it belongs to the space $\bar{K}$, and see (Proposition 8) that such a function has the same properties with respect to Green's theorem as have the functions which vanish on the boundary in the classical case. The statement that a Riemann surface is parabolic means that its boundary is so weak that all functions of class $B D$ have zero boundary values, which in the classical case must mean that the boundary continua have all degenerated to points. Proposition 13 shows that even on an arbitrary (hyperbolic) Riemann surface we can still find the component of a function which has "zero boundary values" simply by forming the integral

$$
f_{K}(q)=\frac{1}{2 \pi} \iint d f * d G
$$

Chapter IV. The space $B D$ on a bounded Riemann surface

18. Some decomposition theorems. On a bounded Riemann surface $V$ we consider the class $B D$ of functions which are piecewise smooth on $V$, bounded, and have differentials belonging to $\Gamma=\Gamma(W)$, where $W$ is the interior of $V$. In addition to the class $K$ of functions having compact carriers it is useful to consider the class $O$ of $B D$ functions which vanish on the boundary $R$ of $V$.

Lemma 18. The linear space $\bar{K} O$ of functions which belong to both $\bar{K}$ and $O$ is closed in the BD topology.

Proof. Since $\bar{K}$ is closed, it is necessary only to show that $O$ is closed. But this is trivial since convergence in $B$ implies uniform convergence on every compact set, whence the limit in $B$ of a function with zero boundary values must again have zero boundary values.

Proposition 17. If $\alpha \in \Gamma$ is a regular differential, and $f \in \bar{K} O$, then

$$
d \alpha[f]=-\iint d f \alpha .
$$

Proof. Since there exists a sequence $\phi_{i}$ of functions in $K O$ whose limit is $f$, we have

$$
\begin{aligned}
d \alpha[f] & =\lim d \alpha\left[\phi_{i}\right] \\
& =-\lim \iint d \phi_{i} \alpha \\
& =-\iint d f \alpha .
\end{aligned}
$$

Theorem 5. On a bounded Riemann surface $V$ the space $B D$ has the decompositions: 


$$
B D=\bar{K} O \oplus H B D=\bar{K} O \oplus H K \oplus H N,
$$

where $H K$ denotes the class of functions belonging to both $H B D$ and $\bar{K}$, and $H N$ the class of those HBD functions whose normal derivative vanishes on $R$. That is to say every $f \in B D$ has the decomposition

$$
f=f_{0}+u_{K}+u_{N}
$$

where $f_{0} \in \bar{K} O, u_{K} \in H K, u_{N} \in H N$, and the decomposition is unique to within a constant. If for $g \in B D$ we set

$$
\|g\|=\sup _{V}|g|
$$

we have $\left\|u_{K}\right\| \leqq\|f\|$ and $\left\|u_{N}\right\| \leqq\|f\|$. Moreover, the decomposition is orthogonal in the sense that

$$
d f=d f_{0}+d u_{K}+d u_{N}
$$

is an orthogonal decomposition.

Proof. Let $\left\{\Omega_{i}\right\}$ be an exhaustion of $V$, and let $u_{i}$ be that piecewise smooth function, guaranteed by Theorem 1 , which is harmonic in $\Omega_{i}$ and identically equal to $f$ outside $\Omega_{i}$. Then as in the proof of Theorem 2 there is a subsequence (which we again call $u_{i}$ ) converging in $B D$ to a harmonic function $u$. Since $f-u_{i} \in K O, f-u$ must belong to $\bar{K} O$ by Lemma 18 . The orthogonality of $d u$ and $d f_{0}=d(f-u)$ follows from Proposition 17.

Hence there remains only to show that $u$ may be split into $u_{K}+u_{N}$. To do this we consider the double $W^{\wedge}$ of $V$ and define $u$ on $W^{\wedge}$ by its symmetric extension. Then by Theorem 2

$$
u=u_{1}+u_{2}
$$

where $u_{1} \in \bar{K}\left(W^{\curlywedge}\right)$ and $u_{2} \in H B D\left(W^{\curlywedge}\right)$. Since symmetrization preserves the classes $\bar{K}\left(W^{-}\right)$and $H B D\left(W^{-}\right)$, we may take $u_{1}$ and $u_{2}$ to be symmetric. Hence $u_{2}$ must have a vanishing normal derivative on $R$ and thus (when restricted to $V$ ) belongs to $H N$. But $u_{1}$ is the $B D$ limit on $W^{\text {人 }}$ of functions of the class $K\left(W^{\wedge}\right)$, whence $u_{1}$ (restricted to $V$ ) is the $B D$ limit of functions of class $K(V)$. Thus $u_{1} \in \bar{K}$, and since $u_{1}=u-u_{2} \in H(V), u_{1} \in H K$. Since $u_{1}$ and $u_{2}$ are symmetric on $W^{\curlywedge}$, and $d u_{1}$ and $d u_{2}$ are orthogonal there, $d u_{1}$ and $d u_{2}$ must also be orthogonal over $V$. This completes the proof.

19. Relative harmonic measures and the type of bounded Riemann surface. We shall say that a bounded Riemann surface $V$ is of (relative) parabolic type if $1 \in \bar{K}(V)$. Otherwise we say that $V$ is of (relative) hyperbolic type. It should be noted that the interior $W$ of $V$ considered as a Riemann surface in its own right is always hyperbolic as soon as the boundary of $V$ is not empty.

Let $\Omega$ be a compact region on $V$ and let $\left\{\Omega_{i}\right\}$ be an exhaustion of $V$ such that $\Omega \subset \Omega_{1}$. In $\Omega_{i}-\Omega$ we construct that piecewise smooth harmonic function 
$u_{i}$ which is identically one on the boundary of $\Omega$, identically zero on the boundary of $\Omega_{i}$, and has a vanishing normal derivative on $R \cap\left(\Omega_{i}-\Omega\right)^{-}$. If we extend the definition of $u_{i}$ so that $u_{i} \equiv 1$ on $\Omega$ and $u_{i} \equiv 0$ outside $\Omega_{i}$, then $u_{i}$ is of class $K$. By the Dirichlet principle $D\left(u_{j}\right) \leqq D\left(u_{i}\right) \leqq D\left(u_{1}\right)$, and by the maximum principle $0 \leqq u_{i} \leqq u_{j} \leqq 1$, for $i<j$. Hence by Lemmas 1 and 2 there is a subsequence which converges together with its derivatives uniformly on every compact set to a harmonic function $u$, which we call the relative harmonic measure of $\Omega$. Clearly, $u$ is independent of the exhaustion chosen, since $u$ may be characterized as the smallest non-negative harmonic function in $V-\Omega$ which is one on $\Omega$ and has zero normal derivative on $R$.

By Green's theorem

$$
D\left(u_{i}-u_{j}, u_{j}\right)=0, \quad i<j,
$$

whence

$$
\begin{aligned}
D\left(u_{i}-u_{j}\right) & =D\left(u_{i}\right)-D\left(u_{j}\right)-2 D\left(u_{i}-u_{i}, u_{j}\right) \\
& =D\left(u_{i}\right)-D\left(u_{i}\right) .
\end{aligned}
$$

Since $D\left(u_{i}\right)$ is a decreasing sequence of positive numbers, it must converge, and so $D\left(u_{i}-u_{j}\right)$ must tend to zero. Hence by Lemma 17

$$
u_{i} \rightarrow u
$$

Thus we have proved the following proposition:

Proposition 18. The relative harmonic measure of a compact region $\Omega$ on a bounded Riemann surface $V$ belongs to the class $\bar{K}$.

THEOREM 6. If $V$ is of hyperbolic type, then every compact region has a nonconstant relative harmonic measure. If $V$ is parabolic, then the relative harmonic measure of each compact region is identically one.

Proof. The first part is a direct consequence of Proposition 18, for if any relative harmonic measure were constant, the constants would belong to $\bar{K}$, making $V$ parabolic. The proof of the second half is identical with the proof of the second part of Theorem 3.

Theorem 7. A necessary and sufficient condition that a bounded Riemann surface be of hyperbolic type is the existence of a regular differential $\alpha \in \Gamma$ such that

$$
d \alpha[\phi]=-\iint d \phi \alpha
$$

for all $\phi \in K$, and

$$
d \alpha[1] \neq 0
$$


Proof. For such a differential

$$
d \alpha[f]=-\iint d f \alpha
$$

for every $f \in \bar{K}$, by the continuity of $d \alpha$ in $B$ and of $\iint d \phi \alpha$ in $D$. Hence if $1 \in \bar{K}$, no such differential can exist.

If on the other hand $V$ is hyperbolic, a compact region must by Theorem 6 have a nonconstant relative harmonic measure $u$. If we set $\alpha=* d u$, then

$$
d \alpha[\phi]=-\iint d \phi \alpha=\int_{\gamma} \phi * d u
$$

by Green's theorem, where $\gamma$ is the boundary of the compact region. Thus $\alpha$ is a regular differential in $\Gamma$, and

$$
d \alpha[1]=\int_{\gamma} * d u=\int_{\gamma} u * d u=D(u) \neq 0,
$$

proving the theorem.

Proposition 19. The space $\bar{K}$ is weakly closed in $B D$.

Proof. By Theorem 5, $f=f_{K}+u_{N}$. But $f_{K}$ is the weak limit of functions of $K$, and hence $u_{N}$ must be a constant $c$, since $d u$ is orthogonal to $\Gamma_{K}$ and hence to $\bar{\Gamma}_{K}$. If the surface $V$ is parabolic, $c \in \bar{K}$, and therefore $f \in \bar{K}$. If the surface is hyperbolic, we choose $\alpha$ by Theorem 7. Now

$$
d \alpha\left[f_{K}\right]=-\iint d f_{K} \alpha
$$

and by weak convergence

$$
d \alpha[f]=-\iint d f \alpha .
$$

Hence

$$
0=-\iint d c \alpha=d \alpha[c]=c d \alpha[1]
$$

and $c=0$, whence $f \in \bar{K}$.

Proposition 20. The space $\bar{K} O$ is weakly closed in $B D$.

Proof. If

$$
f_{i} \rightarrow f
$$

weakly in $B D$

while $f_{i} \in \bar{K} O$, then $f=f_{0}+c$ as before. Let $\alpha$ be a differential which is g.-con- 
tinuous and has a compact carrier and the property that

$$
\int_{R} \alpha=1
$$

Then by Proposition 17

$$
d \alpha\left[f_{i}\right]=-\iint d f_{i} \alpha .
$$

Whence

$$
d \alpha[f]=-\iint d f \alpha
$$

by weak convergence. Also

$$
d \alpha\left[f_{0}\right]=-\iint d f_{0} \alpha .
$$

Therefore

$$
d \alpha[c]=0 .
$$

But

$$
d \alpha[c]=c \int_{R} \alpha=c
$$

whence $c=0$, and the proposition is proved.

Proposition 21. If $V$ is parabolic, then $H N$ contains only constants. Hence $B D=\bar{K}$, and we adopt the convention that $H N$ is empty (i.e., does not even contain constants). Under this convention the decomposition of Theorem 5 is unique.

Proof. Suppose there were a nonconstant $u \in H N$. Then the hypothesis of Theorem 7 is valid with $\alpha=u * d u$, and the surface is hyperbolic.

20. Surfaces with a compact boundary. In this section we assume that $V$ is a bounded Riemann surface whose boundary is compact. If we use the symbol $\dot{+}$ to denote a direct sum, in contrast to $\oplus$ which denotes an orthogonal direct sum, we can then prove the following theorem:

ThEOREM 8. Let $V$ be a bounded Riemann surface with a compact boundary. Then

$$
B D=\bar{K} O+H K+H O=\bar{K}+{ }^{\prime} O .
$$

That is to say if $f \in B D$, then 


$$
f=f_{K}+u_{0}
$$

where $f_{K} \in \bar{K}, u_{0} \in H O$ and the decomposition is unique. Moreover,

$$
\left\|u_{0}\right\| \leqq\|f\| \text {. }
$$

Proof. In view of Theorem 5 we have

$$
\bar{K}=\bar{K} O+H K,
$$

and so it suffices to prove that

$$
B D=\bar{K} \dot{+} H O .
$$

Let $\left\{\Omega_{i}\right\}$ be an exhaustion of $V$ such that $R \subset \Omega_{1}$, and $R$ separated from the remainder of the boundary of $\Omega_{1}$. Let $f_{1}$ be a piecewise smooth function which is identically equal to $f$ outside $\Omega_{1}$, and vanishes on $R$. Set $u_{i}$ equal to that piecewise smooth function given by Theorem 1 which vanishes on $R$, is harmonic in $\Omega_{i}$, and is identically equal to $f\left(=f_{1}\right)$ outside $\Omega_{i}$. Then

$$
0 \leqq D\left(u_{i}\right) \leqq D\left(u_{i}\right) \leqq D\left(f_{1}\right)
$$

for $i<j$. Also

$$
\boldsymbol{u}_{i} \leqq \sup _{p \in \Omega_{j}}|f(p)|=\sup _{p \in V}|f(p)| .
$$

Hence by Harnack's principle there is a subsequence (which we again call $u_{i}$ ) of functions which converge together with their derivatives to a harmonic function $u_{0}$ which vanishes on $R$, the convergence being uniform on every compact set. Thus by Lemma 17

$$
u_{i} \rightarrow u_{0}
$$

in $B D$.

Since $f-u_{i} \in K, f-u_{0} \in \bar{K}$, and it remains only to prove that the decomposition (2) is unique. But this is certainly true, for if $u \in H O$ and $u \in \bar{K}$, then $u \in H B D$ and $u \in \bar{K} O$, whence, by Theorem $5, u$ must be a constant, and the constant must be zero since $u$ vanishes on $R$.

THEOREM 9. On a bounded Riemann surface with a compact boundary there is a natural algebraic isomorphism $\pi_{0}$ between the spaces $H N$ and $H O$ (provided we adopt the convention of Proposition 21 concerning constants). Moreover $\|u\|=\left\|\pi_{0} u\right\|$.

Proof. The algebraic isomorphism follows from Theorems 5 and 8 since $H N$ and $H O$ are both isomorphic to the quotient space $B D / \bar{K}$. In order to show that the norms are preserved, however, we shall actually construct $\pi_{0}$.

If $u \in H N$, then the decomposition of Theorem 8 gives

$$
u=u_{0}+u_{K},
$$

where $u_{0} \in H O$ and $u_{K} \in \bar{K}$, with $\left\|u_{0}\right\| \leqq\|u\|$. But by the uniqueness of the 
decompositions in Theorems 5 and 9 , we must have for $u_{0}$ the decomposition

$$
u_{0}=u-u_{K}
$$

with $\|u\| \leqq\left\|u_{0}\right\|$ by Theorem 5 .

Thus the mapping $\pi_{0}: u \rightarrow u_{0}$ is one-to-one isometric onto, and is easily seen to be linear, thus proving the theorem.

At this point I consider it a reasonable conjecture that $\pi_{0}$ is actually bicontinuous in the $B D$ topology. However, this seems to be a difficult thing to prove because of our meager knowledge of the $B D$ topology.

It should be noted that $\pi_{0} 1=1-u$ where $u$ is the harmonic measure of $R$ with respect to $V$, for it is easily proved that $u \in \bar{K}$. In view of Theorem 9 we have the following characterization of the type of a bounded Riemann surface $V$ with a compact boundary:

Proposition 22. A bounded Riemann surface $V$ with a compact boundary $R$ is of (relative) hyperbolic type if and only if the harmonic measure of $R$ with respect to $V$ is not constant.

In [25] there are given counterexamples which show that the results of this section are no longer valid if we do not require $V$ to have a compact boundary.

\section{Separations by a compact set of curves.}

THEOREM 10. Let $W$ be an open Riemann surface, and let $R$ consist of a finite number of closed analytic curves which separate $W$ into the regions $W_{i}, i=1, \cdots$, $N$. Take $V_{i}$ to be the bounded Riemann surface whose interior is $W_{i}$ and whose boundary is $R_{i}=R \cap W_{i}$. Then under the convention of Proposition 21 there are algebraic isomorphisms $\pi_{1}$ and $\pi_{2}$ which map $H B D(W)$ onto

$$
H O\left(V_{1}\right)+\cdots+H O\left(V_{N}\right)
$$

and onto

$$
H N\left(V_{1}\right)+\cdots+H N\left(V_{N}\right)
$$

respectively. Moreover

$$
\|u\|=\left\|\pi_{1} u\right\|=\left\|\pi_{2} u\right\| .
$$

Proof. In view of Theorem 9 it suffices to prove the statements for $\pi_{1}$. We first note that a function $\phi$ which is piecewise smooth on $W$ has a compact carrier if and only if the restrictions of $\phi$ to each of the $V_{i}$ each have compact carriers. Consequently, a function $f$ of class $B D$ on $W$ belongs to $\bar{K}(W)$ if and only if the restrictions of $f$ to $V_{i}$ each belong to $\bar{K}\left(V_{i}\right)$.

If we use $H O(W)$ to denote those $B D$ functions on $W$ which are harmonic in $W-R$ and vanish on $R$, then clearly $H O(W)$ is isomorphic to $H O\left(V_{1}\right)$ $\dot{+} \cdots+H O\left(V_{N}\right)$ in a natural way which preserves norms. But in Theorem 8 we see that $f \in B D$ has the unique decomposition 


$$
f=f_{K}+u_{0}
$$

where $f_{K} \in \bar{K}(W)$ and $u_{0} \in H O(W)$, with $\left\|u_{0}\right\| \leqq\|f\|$. Also a function $f \in B D$ has the unique decomposition

$$
f=f_{K}^{\prime}+u
$$

with $f_{K}^{\prime} \in \bar{K}(W)$ and $u \in H B D(W)$ with $\|u\| \leqq\|f\|$. Thus for $u \in H B D(W)$ we have by (3) the unique decomposition

$$
u=u_{K}+u_{0}
$$

with $\left\|u_{0}\right\| \leqq\|u\|$. But by (4) we have

$$
u_{0}=-u_{K}+u
$$

with $\|u\| \leqq\left\|u_{0}\right\|$, where $u_{K}$ and $u$ must be the same as in (5) by the uniqueness of the decompositions (3) and (4). Thus $\left\|u_{0}\right\|=\|u\|$, and $\pi_{1}: u \rightarrow u_{0}$ is an isometric isomorphism onto.

22. The unrestricted maximum principle and the character of a bounded Riemann surface. We say that the unrestricted maximum principle does not hold on a bounded Riemann surface $V$, or that $V$ is of hyperbolic character, if there is a real bounded harmonic function $u$ which is nonpositive on the boundary $R$ of $V$ and which is positive somewhere in $V$. We shall say that the unrestricted maximum principle does not hold on an open Riemann surface $W$ if there is at least one subregion of $W$ on which the unrestricted maximum principle does not hold.

Propositron 23. A necessary and sufficient condition that a Riemann surface $W$ be hyperbolic is that the unrestricted maximum principle does not hold on $W$.

Proof. By Theorem 3 there exists a compact region $\Omega$ which has a nonconstant harmonic measure $u$. The harmonic function $1-u$ violates the unrestricted maximum principle in $W-\Omega$.

Suppose on the other hand that there is a subregion $W_{1} \subset W$ in which the unrestricted maximum principle does not hold. We may assume a compact region $\Omega \subset W-W_{1}$ since the validity of the unrestricted maximum principle is not affected by the addition or subtraction of a compact region. Let $v$ be a real bounded harmonic function in $W_{1}$ which is nonpositive on the boundary $R$ of $W_{1}$ and positive somewhere in $W_{1}$. We normalize $v$ so that it is bounded by unity. Hence by the maximum principle for compact regions we have $u_{i} \leqq 1-v$, where $u_{i}$ is the sequence of harmonic measures used to define the harmonic measure $u$ of the region $\Omega$. Hence $u \leqq 1-v$, and $u$ is not constant since $1-v<1$ at some points of $W_{1}$. Thus $W$ is hyperbolic by Theorem 3 .

Proposition 24. A necessary and sufficient condition that a Riemann surface $W$ have a nonconstant bounded harmonic function on it is the existence on it of two disjoint subregions of hyperbolic character. 
The proof is as in [24].

Proposition 25. $A$ bounded Riemann surface $V$ whose boundary $R$ is compact is of hyperbolic character if and only if it is of hyperbolic type.

Proof. If it is of hyperbolic character, let $v$ with $v<1$ be nonpositive on $R$ and positive somewhere in $V$. Then if we define the harmonic measure $u$ of $R$ with respect to $V$ as the limit of the harmonic measures $u_{i}$ in an exhaustion of $V$, we have $u_{i} \leqq 1-v$, whence $u \leqq 1-v$. Thus $u$ is not identically one, since $1-v$ is somewhere less than one. Consequently, $V$ is of hyperbolic type by Proposition 22.

If on the other hand $V$ is of hyperbolic type, then by Proposition 22 the boundary $R$ must have a nonconstant harmonic measure $u$ with respect to $V$, and $1-u$ violates the unrestricted maximum principle.

Proposition 26. If a bounded Riemann surface $V$ has a compact boundary $R$, then the existence of a nonconstant bounded harmonic function with a vanishing normal derivative on $R$ implies that $V$ is of hyperbolic character.

Proof. A harmonic function with a vanishing normal derivative on $R$ can take neither its maximum nor its minimum on $R$ if it is not constant. Hence such a function violates the unrestricted maximum principle if it is bounded.

Counterexamples are given in [25] which show that the restrictions to compact boundaries are essential.

23. The classification of Riemann surfaces. If we denote the class of parabolic surfaces by $N_{G}$ and the class of Riemann surfaces on which there are no nonconstant positive harmonic functions by $N_{H P}$, then I maintain

$$
N_{G} \subseteq N_{H P}
$$

For let $v$ be a nonconstant positive harmonic function on a Riemann surface $W$, and assume that the greatest lower bound of $v$ is zero. Then a component $W_{1}$ of the set where $v<1$ is a subregion in which $1-v$ violates the unrestricted maximum principle, and hence $W$ is hyperbolic by Proposition 23.

Clearly

$$
N_{H P} \subseteq N_{H B},
$$

since the real part of a bounded harmonic function becomes a positive harmonic function by the addition of a suitable constant.

By virtue of Proposition 16 we have

$$
N_{H D}=N_{H B D} .
$$

Hence we may summarize these relations in the following proposition:

Proposition 27. We have 


$$
N_{G} \subseteq N_{H P} \subseteq N_{H B} \subset N_{H B D}=N_{H D} .
$$

In [25] an example is given which shows that the inclusion of $N_{H B}$ in $N_{H D}$ is strict. In [24] it was shown that for analytic functions

$$
N_{A B} \subset N_{A D} \text {. }
$$

It is known [9] that $N_{A B}$ is not contained in $N_{H D}$, but it is not known whether or not the inclusion holds in the opposite direction. In the "schlichtartig" case

$$
N_{G}=N_{H D}
$$

and these surfaces are characterized by being mapped onto plane regions whose complements have logarithmic capacity zero.

The characterization of $N_{G}$ by means of the maximum principle is due to Myrberg [15]. The inclusion of $N_{H B}$ in $N_{H D}$ was shown by Virtanen [35]. For the "schlichtartig" case see [9] and [15].

\section{Chapter V. Some applications to The type PRoblem}

24. Triangulation of a Riemann surface. By a polygon $s^{2}$ on a Riemann surface $W$ we mean a pair $\left(S^{2}, G\right)$ consisting of a polygon $S^{2}$ in the plane and a mapping $G$ which is a conformal mapping of the interior of $S^{2}$ into $W$ which satisfies the following conditions:

(i) On (the interior of) each edge of $S^{2}$ the mapping $G$ is analytic and oneto-one.

(ii) At a vertex $S^{0}$ of $S^{2}$ either $G$ is analytic or else for any compact region $\Omega_{0} \subset W$ there is a neighborhood $U$ of $S^{0}$ such that $G(U)$ does not meet $\Omega_{0}$.

Thus, roughly speaking, a polygon on a Riemann surface is the conformal image of a Euclidean polygon. However, some of the vertices of the Euclidean polygon may not be mapped into interior points of $W$, but rather so to say onto the boundary of $W$. The images under $G$ of the interior, edges, and vertices of $S^{2}$ will be called the interior, edges, and vertices, respectively, of $s^{2}$. Thus the edges of $s^{2}$ are analytic arcs, which may, however, overlap one another. The points in the interior, edges, and vertices of $s^{2}$ are the points of $s^{2}$.

A triangulation of $W$ is a collection of polygons $s_{i}^{2}=\left(S_{i}^{2}, G_{i}\right)$ such that:

(i) Every point $p \in W$ is a point of some polygon $s_{i}^{2}$.

(ii) If a point $p \in W$ is an interior point of some $s_{\imath}^{2}$, then it belongs to no other polygon.

- (iii) If a point $p \in W$ belongs to an edge of some $s_{i}^{2}$, then it belongs to exactly one other edge (which may belong to $s_{t}^{2}$ or to some other polygon).

That we may take the indices to run over the integers is a consequence of the fact that a Riemann surface is separable.

The fact that we have not required the vertices of a triangulation to be points of the Riemann surface has the advantage that if we have a triangulation of a Riemann surface $W$, it is also a triangulation of the Riemann surface 
$W^{\prime}$ formed by removing one or more of the points of $W$ which are vertices of the triangulation. Also we have allowed two edges of a single polygon to meet, which has the advantage that any compact Riemann surface $W$ (as well as the surface $W^{\prime}$ formed by removing a finite number of points from $W$ ) can be triangulated with a single polygon, e.g., by making a finite number of analytic cuts which reduce the surface to a simply-connected one and mapping this onto a suitable plane polygon.

For our purposes a two-dimensional cochain $A^{2}$ on a triangulation may be defined as a mapping of the polygons of the triangulation into the real numbers. We shall write

$$
A^{2}=\sum_{i=1}^{\infty} a_{i}^{2} s_{i}^{2}
$$

where $a_{i}^{2}$ is the value of the mapping on $s_{i}^{2}$. If a polygon $s_{j}^{2}$ does not appear in the formal sum (1), then we mean that $a_{j}^{2}=0$.

A one-dimensional cochain $A^{1}$ on a triangulation is a mapping of the edges of the Euclidean polygons $S_{i}^{2}$ into the real numbers having the property that two edges which have a common image on the Riemann surface are mapped into numbers which are the negatives of one another. We define an oriented edge $s_{i}^{1}$ of the triangulation to be a pair $\left(S_{f}^{1}, S_{k}^{1}\right)$ of edges of Euclidean polygons $S_{j}^{2}$, and $S_{k}^{2}$, which have the same image on the Riemann surface, and write

$$
\left(S_{j}^{1}, S_{k}^{1}\right)=-\left(S_{k}^{1}, S_{j}^{1}\right)
$$

Then we may write

$$
A^{1}=\sum_{i=1}^{\infty} a_{i}^{11} s_{i}
$$

where only one of the pairs $s_{i}^{1},-s_{i}^{1}$ appears in the sum, and $a_{i}^{1}$ is the value of the cochain on the first element of $s_{i}^{1}$.

The $p$-dimensional cochains ( $p=1$ or 2 ) form an Abelian group under the definition

$$
A^{p}+B^{p}=\sum_{i=1}^{\infty}\left(a_{i}^{p}+b_{i}^{p}\right) s_{i}^{p}
$$

We may define a homomorphism $\delta$ of the group of one-dimensional cochains as follows: If $s^{1}=\left(S_{y}^{1}, S_{k}^{1}\right)$,

$$
\delta s_{i}^{1}=s_{j^{\prime}}^{2}-\underset{s_{k^{\prime}}}{2},
$$

whese $s_{j^{\prime}}^{2}$ is the polygon such that $S_{j}^{1}$ is an edge of $S_{j^{\prime}}^{2}$, and $s_{k^{\prime}}^{2}$ the polygon such that $S_{\mathfrak{k}}^{1}$ is an edge of $S_{\mathfrak{k}^{2}}^{2}$. On $A^{1}$ we define $\delta$ by 


$$
\delta A^{1}=\sum_{i=1}^{\infty} a_{i}^{1} \delta s_{i}^{1}
$$

25. The $\beta$-measure of a polygon. Let $S$ be an $n$-sided polygon in the plane, and let $\gamma$ be a differential which is defined and continuous on $S$, except possibly at the vertices of $S$. Suppose that $\left\{m_{k}\right\}$ is a set of $n$ real numbers whose sum is zero.

If $\gamma$ is (absolutely) integrable along each edge $C_{k}$ of $S$ (note that $\gamma$ need be neither defined nor continuous at the end points of $C_{k}$ ), and if

$$
I_{k}=\int_{C_{k}} \gamma \neq 0, \quad k=1, \cdots, n,
$$

we can construct a function $v=v\left(m_{1}, \cdots, m_{n} ; \gamma\right)$ which is harmonic in $S$ and which satisfies

$$
* d v=m_{k} \gamma / I_{k} \quad \text { along } C_{k} \text {, }
$$

and consequently

$$
\int_{C_{k}} * d v=m_{k}
$$

Indeed, to prescribe $* d v$ along $C_{k}$ is merely to prescribe $\partial v / \partial n$ on $C_{k}$. In order to infer the existence of $v$ by the classical solution of the Neumann problem one has only to observe that

$$
\int_{\Sigma C_{k}} \frac{\partial v}{\partial n}=\sum m_{k}=0 .
$$

It is now possible to define a measure $\lambda_{\gamma}(S)$ of the polygon with respect to the differential $\gamma$. We set $\lambda_{\gamma}(S)$ equal to the maximum for all sets $\left\{m_{k}\right\}$ satisfying

$$
\sum m_{k}=0, \quad \sum\left|m_{k}\right|^{2}=1
$$

of the Dirichlet integral of $v\left(m_{1}, \cdots, m_{k} ; \gamma\right)$, provided $v\left(m_{1}, \cdots, m_{n} ; \gamma\right)$ can be defined for all $m_{k}$ satisfying (7). If not, e.g., if the integral of $\gamma$ over some $C_{k}$ vanishes or if $\gamma$ is not absolutely integrable over some $C_{k}$, we set $\lambda_{\gamma}(S)=\infty$.

As a consequence one has the inequality

$$
D_{S v}\left(m_{1}, \cdots, m_{n} ; \gamma\right) \leqq \lambda_{\gamma}(S) \sum_{k=1}^{n}\left|m_{k}\right|^{2},
$$

with the function $v\left(m_{1}, \cdots, m_{n} ; \gamma\right)$ existing whenever the right-hand side of (8) is finite. For 


$$
D_{S}(v)=c^{2} D_{S}\left(\frac{1}{c} v\right),
$$

and we need only take $c^{2}=\sum m_{\mathrm{k}}^{2}$. In the right-hand side of (8) we may adopt the convention that $0 \cdot \infty=0$, since

$$
v(0, \cdots, 0 ; \gamma)=0 \text {. }
$$

This definition of measure can be extended to a polygon

$$
s_{i}^{2}=\left(S_{i}^{2}, G\right)
$$

and a differential $\beta$ on $W$ by setting

$$
\lambda_{\beta}\left(s_{i}^{2}\right)=\lambda_{G^{*} \beta}\left(S_{i}^{2}\right) .
$$

Thus the $\beta$-measure of a polygon on a Riemann surface is unchanged by a conformal mapping provided the differential $\beta$ undergoes the adjoint mapping.

The function $v=v\left(m_{1}, \cdots, m_{n} ; G^{*} \beta\right)$ on $S_{i}^{2}$ can be extended to a harmonic function $u=u\left(m_{1}, \cdots, m_{n} ; \beta\right)$ defined on the image of $S_{i}^{2}$ by setting

$$
u=G_{i}^{-1 *} v
$$

It is then clear that we have

$$
\int_{G C_{k}} * d u=m_{k}
$$

and

$$
D(u) \leqq \lambda_{\beta}\left(s_{i}^{2}\right) \sum_{k=1}^{n}\left|m_{k}\right|^{2},
$$

the function $u$ being defined whenever the right-hand side is finite (with the convention still that $0 \cdot \infty=0$ ).

We define the $\beta$-measure of an edge of a triangulation to be the sum of the $\beta$-measures of the two polygons of which it is an edge.

With this in mind we define the $\beta$-norm of a one-dimensional cochain

$$
A^{1}=\sum_{i=1}^{\infty} A_{i}^{1} s_{i}^{1}
$$

as

$$
N_{\beta}\left(A^{1}\right)=\sum_{i=1}^{\infty}\left|a_{i}^{1}\right|^{2} \lambda_{\beta}\left(s_{i}^{1}\right)
$$

with the convention that $0 \cdot \infty=0$. 
26. A condition for a Riemann surface to be hyperbolic.

THEOREM 11. A sufficient condition that a Riemann surface be hyperbolic is the existence of a triangulation for which there is a cochain $A^{1}$ and a differential $\beta$ such that

$$
N_{\beta}\left(A^{1}\right)<\infty
$$

and

$$
\delta A^{1}=s_{0}^{2},
$$

where $s_{0}^{2}$ is a polygon of the triangulation.

Proof. Let $A^{1}=\sum a_{i}^{1} s_{i}^{1}$. We shall define a differential as follows: Let $s_{t_{k}}^{1}$ be the edges of an $n$-sided polygon $s_{j}^{2}, j \neq 0$. Then we set $m_{k}= \pm a_{t_{k}}^{1}$ according to whether the first or second element of $s_{t_{b}}^{1}$ is an edge of $S_{j}^{2}$. Since $s_{j}^{2}$ does not appear in the expression for $A^{1}$, we must have

$$
\sum_{k=1}^{n} m_{k}=0
$$

Since also

$$
\lambda_{\beta}\left(s_{j}^{2}\right) \sum_{k=1}^{n}\left|m_{k}\right|^{2} \leqq \sum_{k=1}^{n} \lambda_{\beta}\left(s_{j_{k}}^{1}\right)\left|a_{i_{k}}^{1}\right|^{2} \leqq N\left(A^{1}\right),
$$

we have $u=u\left(m_{1}, \cdots, m_{n} ; \beta\right)$ defined and we set

$$
\alpha=* d u
$$

in the interior of $s_{j}^{2}$.

On $S_{0}^{2}$ we define $\alpha$ by setting it equal to an arbitrary continuously dif. ferentiable differential which is a constant multiple of $\beta$ along each of the $s_{t_{k}}^{1}$ which is an edge of $s_{0}^{2}$ and requiring

$$
\int_{\substack{\boldsymbol{s}_{i k}^{1} \\ i_{k}}} \alpha=m_{k}= \pm a_{i k}^{1}
$$

as before. Clearly

$$
\int_{s_{0}^{2}} \alpha * \bar{\alpha}=\sum_{k=1}^{n} m_{k}=1
$$

since $s_{0}^{2}$ is the coboundary of $A^{1}$.

Now

$$
\|\alpha\|^{2}=\iint_{i_{0}^{2}} \alpha * \bar{\alpha}+\sum_{j=1}^{\infty} D_{j}^{2} u\left(m_{1}, \cdots, m_{n_{j}} ; \beta\right) .
$$


By inequality (12)

$$
\|\alpha\|^{2} \leqq \iint_{i_{0}^{2}} \alpha * \bar{\alpha}+\sum_{j=1}^{\infty} \sum_{k=1}^{n_{j}}\left|a_{i_{k}}^{1}\right|^{2} \lambda_{\beta}\left(s_{j}^{2}\right),
$$

where $s_{i_{k}}^{1}$ are the edges of $s_{j}^{2}$. Since $\lambda_{\beta}\left(s_{j}^{2}\right) \leqq \lambda_{\beta}\left(s_{t_{k}}^{1}\right)$, we have

$$
\|\alpha\|^{2}=\iint_{s_{0}^{2}} \alpha * \bar{\alpha}+\sum_{j=1}^{\infty} \sum_{k=1}^{n_{j}}\left|a_{i_{k}}^{1}\right|^{2} \lambda_{\beta}\left(s_{i_{k}}^{1}\right) .
$$

Since each $s_{k_{k}}^{1}$ occurs in this sum at most twice,

$$
\|\alpha\|^{2} \leqq \iint_{\delta_{0}^{2}} \alpha * \bar{\alpha}+2 \sum_{i=1}^{\infty}\left|a_{i}^{1}\right|^{2} \lambda_{\beta}\left(s_{i}^{1}\right) \leqq \iint_{\delta_{0}^{2}} \alpha * \bar{\alpha}+2 N\left(A^{1}\right) .
$$

Thus $\alpha \in \Gamma$.

Now $\alpha$ is a piecewise continuous and piecewise continuously differentiable differential which has the edges $s_{i}^{1}$ as its only lines of discontinuity. But along $s_{i}^{1}$ we have

$$
\alpha=\frac{a_{i}^{1} \beta}{\int_{\varepsilon_{i}^{1}}^{1} \beta}
$$

no matter which side we approach $s_{i}^{1}$ from. Thus $\alpha$ is g.-continuous and by Lemma 10 we have

$$
d \alpha[f]=\iint_{\delta_{0}^{2}} f d \alpha
$$

and so $\alpha$ is regular. But

$$
d \alpha[1]=\iint_{\delta_{0}^{2}} d \alpha=1 \neq 0 .
$$

Hence $W$ is hyperbolic by Theorem 4. This completes the proof of our theorem.

If a triangulation $\left\{s_{t}^{2}\right\}$ has the property that there exists a differential $\beta$ such that

$$
\lambda_{\beta}\left(s_{i}^{2}\right)<M
$$

for all $i$, then we call the triangulation uniform. We shall define the norm of a one-dimensional cochain to be

$$
N\left(A^{1}\right)=\sum_{i=1}^{\infty}\left|a_{i}^{1}\right|^{2}
$$


For a uniform triangulation we have

$$
N_{\beta}\left(A^{1}\right) \leqq 2 M N\left(A^{1}\right) .
$$

Hence we have the following important corollary to Theorem 11:

COROLlaRY. If $\left\{s_{i}^{2}\right\}$ is a uniform triangulation on a Riemann surface $W, W$ is hyperbolic if there is a one-dimensional cochain $A^{1}$ on the triangulation $\left\{s_{t}^{2}\right\}$ such that

$$
N\left(A^{1}\right)<\infty
$$

and

$$
\delta A^{1}=\stackrel{2}{s_{0}} .
$$

27. The type of a covering surface. Let $W^{\prime}$ be a Riemann surface formed by removing a finite number of points from a compact surface. Then as noted before $W^{\prime}$ may be triangulated by a single polygon $s^{2}=\left(S^{2}, G\right)$.

A covering surface $(\tilde{W}, F)$ for an open Riemann surface $W^{\prime}$ is a pair consisting of a Riemann surface $\tilde{W}$ and an analytic mapping $F$ of $\tilde{W}$ onto $W^{\prime}$ with the proper'y that every point of $W^{\prime}$ has a neighborhood $U$ in which $F^{-1}$ is a homeomorphism onto each component of $F^{-1}(U)$. It can be shown that this property holds true provided $U$ is any simply-connected region on $W^{\prime}$. Hence it is readily seen that a triangulation of $W^{\prime}$ gives rise to a triangulation of $\tilde{W}$ for which $F$ is a homeomorphism on each polygon onto a polygon of the triangulation for $F$.

Let $\beta$ be a continuous differential on $W^{\prime}$ which has the property that the integral of $\beta$ along each of the edges of $s^{2}$ exists and does not vanish. Such a differential is easily constructed since the edges of $s^{2}$ are analytic arcs. For such a differential it is clear that the functions

$$
\begin{aligned}
v_{1} & =v(1,0, \cdots, 0,-1 ; \beta), \\
v_{2} & =v(0,1, \cdots, 0,-1 ; \beta), \\
. & . . . . . . \cdot, \\
v_{n-1} & =v(0,0, \cdots, 1,-1 ; \beta)
\end{aligned}
$$

all exist and have a finite Dirichlet integral which we denote by $D_{i}$. Since

$$
v\left(m_{1}, \cdots, m_{n} ; \beta\right)=\sum_{i=1}^{n-1} m_{i} v_{i}
$$

for $\sum m_{i}=0$, we have on applying Minkowski's inequality

$$
[D(v)]^{1 / 2} \leqq \sum_{i=1}^{n-1}\left|m_{i}\right|\left[D_{i}\right]^{1 / 2} \leqq D^{1 / 2} \sum_{i=1}^{n}\left|m_{i}\right|,
$$

where we have set $D$ equal to the largest of the $D_{i}$. By the Schwarz inequality 


$$
D(v) \leqq n D \sum_{i=1}^{n}\left|m_{i}\right|^{2}
$$

Thus

$$
\lambda_{\beta}\left(s^{2}\right) \leqq n D<\infty .
$$

If we set $\widetilde{\beta}=F^{*} \beta$, then

$$
\lambda_{\beta}\left(s_{i}^{2}\right)=\lambda_{\beta}\left(s^{2}\right)
$$

by the invariance of the $\beta$-measure under conformal mapping. Thus the triangulation $\left\{s_{i}^{2}\right\}$ is uniform and the corollary to Theorem 11 becomes Theorem 12:

THEOREM 12. Let $(\tilde{W}, F)$ be a covering surface of a Riemann surface $W^{\prime}$ obtained by removing a finite number of points from a compact Riemann surface. Let $\left\{s_{\imath}^{2}\right\}$ be a triangulation of $W$ arising from a triangulation of $W^{\prime}$ by a single polygon. Then a sufficient condition that $W$ be of hyperbolic type is the existence of a one-dimensional cochain $A^{1}$ on $\left\{s_{i}^{2}\right\}$ such that

$$
N\left(A^{1}\right)<\infty
$$

and

$$
\delta A^{1}=\stackrel{2}{s_{0}}
$$

The reader should have no difficulty in generalizing this theorem to triangulations $\left\{s_{i}^{2}\right\}$ of $W$ which arises from any finite triangulation of $W^{\prime}$.

28. The Speiser linear graph. Let $\left\{s_{i}^{2}\right\}$ be a triangulation of a covering surface $(\tilde{W}, F)$ arising from the triangulation of the base surface $W^{\prime}$ by a a single polygon. We may then construct a linear graph dual to the triangulation in the following way: Let $t_{0}^{i}$ be a point in the interior of $s_{i}^{2}$ for each $i$. If two polygons $s_{i}^{2}$ and $s_{f}^{2}$ have an edge $s_{k}^{1}$ in common, we join $t_{0}^{i}$ and $t_{0}^{j}$ by an analytic arc $t_{1}^{k}$ lying entirely in the interiors of $s_{i}^{2}, s_{j}^{2}$, and $s_{k}^{1}$. If, say,

$$
\delta s_{k}^{1}=\stackrel{2}{s_{i}}-\stackrel{2}{s_{j}}
$$

we orient the arc $t_{1}^{k}$ so that its boundary satisfies the relation

$$
\partial t_{1}^{k}=t_{0}^{i}-t_{0}^{j}
$$

This linear graph is known as the Speiser linear graph (Streckenkomplex) of $W$, and has been considered in some detail by R. Nevanlinna [19]. By duality Theorem 12 becomes Theorem 12':

TheOREM 12'. A sufficient condition that a covering surface $(\tilde{W}, F)$ of a Riemann surface $W^{\prime}$ obtained by removing a finite number of points from a compact Riemann surface be hyperbolic is that there be a one-dimensional chain 


$$
A_{1}=\sum_{i=1}^{\infty} a_{1}^{i} t_{1}^{i}
$$

on the linear graph of $\tilde{W}$ with the properties that

$$
\partial A_{1}=\stackrel{0}{t_{0}^{0}}
$$

and

$$
N\left(A_{1}\right)=\sum_{i=1}^{\infty}\left|a_{1}^{i}\right|^{2}<\infty .
$$

29. Covering surfaces of type $S$. By a circular domain is meant a plane region bounded by a finite number of disjoint circles $C_{k}, k=1, \cdots, n$. If a circular domain is considered as a sort of generalized polygon with the sides $C_{k}$ and without vertices, it is possible in some cases to define a generalized triangulation of a Riemann surface $W$ by the conformal images of circular domains. In particular if $W$ is a compact Riemann surface, it is possible to construct a generalized triangulation of $W$ by means of a single generalized polygon, e.g., by making $W$ into a surface of genus zero ("schlichtartig") by a finite number of disjoint cuts and mapping this surface onto the exterior of a finite number of circles. Since the generalized polygon is no longer simply-connected, we can no longer infer that such a triangulation induces a triangulation of an arbitrary covering surface of $W$.

This leads us to consider covering surfaces of $W$ with the property that there is a generalized triangulation on them which arises from a triangulation of the base surface (which must necessarily be compact) by means of a single generalized polygon. Such covering surfaces we shall call covering surfaces of type $S$. Clearly we may extend the notion of cochains to generalized triangulations of such surfaces.

PROPOSITION 28. In order that a covering surface of type $S$ be hyperbolic it is necessary and sufficient that the generalized triangulation arising from the triangulation of the base surface by a single circular domain has on it a onedimensional cochain $A^{1}$ so that

$$
\delta A^{1}=s_{0}^{2}
$$

and

$$
N\left(A^{1}\right)<\infty .
$$

Proof. The sufficiency of the condition is proved in exactly the same manner as Theorem 12. On the other hand suppose that there is a Green's function on the covering surface $W$ with a pole in the interior of the polygon $s_{0}^{2}$. To each edge $s_{\mathbf{k}}^{1}$ of the triangulation we attach the value 


$$
a_{k}^{1}=\frac{1}{2 \pi} \int_{\delta_{k}^{1}} * d G
$$

where the direction of integration on $s_{k}^{1}$ is such that the interior of $s_{i}^{2}$ is to the right where $s_{k}^{1}=s_{j}^{2}-s_{i}^{2}$. Now

$$
A^{1}=\sum_{i=1}^{\infty} a_{i}^{1} s_{i}
$$

is clearly a cochain on the triangulation. Since the integral of $* d G$ over the boundary of any polygon not containing the pole of the Green's function vanishes, we have

$$
\delta A^{1}=\stackrel{2}{s_{0}}
$$

Hence to complete the proof of the theorem it need only be shown that

$$
\sum_{i=1}^{\infty}\left|a_{i}^{1}\right|^{2}<\infty
$$

Let $R$ be the circular domain in the plane whose images by the functions $G_{i}$ are the polygons $s_{i}^{2}$. Then the function $G_{i}^{*} G$ is a harmonic function in the interior of $R$, and the numbers $a_{f_{k}}^{1}$ representing the integrals of $* d G_{t}^{*} G$ over the boundary circles $C_{k}$ of $R$ are nothing more than the periods of * $d G_{i}^{*} G$ over the different homology classes of $R$. Let $m$ be the maximum of the numbers $\left|a_{j_{k}}^{1}\right|$. Then the norm in $R$ of a harmonic differential which has at least one period larger than $m$ cannot become arbitrarily small by Proposition $7^{\prime}$, and we denote the smallest possible norm by $M(m)$. From the quadratic nature of the norm we have

$$
M(m)=m^{2} M(1)
$$

with $M(1)>0$. Let $R$ have $n$ boundaries. Then we have

$$
\begin{aligned}
\|d G\|_{\delta_{i}^{2}} & =\left\|* d G_{i}^{*} G\right\|_{R} \\
& \geqq M(m)=m^{2} M(1) \\
& \geqq \frac{1}{n} M(1) \sum_{k=1}^{n}\left|a_{j_{k}}^{1}\right|^{2} .
\end{aligned}
$$

Thus

$$
\sum_{i=1}^{\infty}\|d G\|_{\delta_{i}^{2}} \geqq \frac{M(1)}{n} \sum_{j=1}^{\infty}\left|a_{j}^{1}\right|^{2} .
$$

But the left-hand side is just the Dirichlet integral of $G$ over the exterior of the polygon $s_{0}^{2}$ and is thus finite. Hence 


$$
\sum_{i=1}^{\infty}\left|a_{i}^{1}\right|^{2}<\infty
$$

and we have proved the proposition.

The method of proof also yields the following interesting corollary:

Corollary. If we define

$$
\pi u=A_{u}^{1}=\sum_{j=1}^{\infty} a_{j}^{1} s_{j}^{1}
$$

where

$$
a_{j}^{1}=\int_{\substack{s_{j}^{1} \\ j_{j}}} * d u,
$$

then $\pi$ is an isomorphism of the class $H D$ onto the group of cocycles of finite norm. Moreover, there exist two nonzero constants $N_{1}$ and $N_{2}$ such that

$$
N_{1} D(u) \leqq N\left(A_{u}^{1}\right) \leqq N_{2} D(u)
$$

for all $u$ belonging to the class $H D$.

30. The Schottky covering surface. A covering surface of type $S$ which is of genus zero is called a Schottky covering surface. For a covering surface of type $S$ to have genus zero it is necessary and sufficient that there be no finite cycles in the Speiser linear graph of $W$, for such a cycle in the graph corresponds to a compact cycle on $W$ which intersects just once some of the edges of a generalized triangulation of $W$.

Starting from a point $t_{0}^{0}$ of the graph, we call the segments emanating from it first generation segments and their remote (i.e., other than $t_{0}^{0}$ ) end points first generation points. In general we call segments which are not of the $(n-1)$ st generation, but which emanate from points of the $n$th generation, $n$th generation segments and their remote end points $(n+1)$ st generation points. Since there are no finite cycles in the graph, it follows that each point and each segment of the graph belong to a unique generation. If the base surface $W$ of $\tilde{W}$ has genus $p \geqq 1$, then $p$ cuts are required to reduce $W$ to a surface of genus zero, and hence a circular domain which effects a generalized triangulation of $W$ has $2 p$ disjoint circles for boundaries. Thus $2 p$ segments . emanate from each point of the graph, and there are $2 p(2 p-1)^{n-1}$ segments in the $n$th generation.

If we assume the $n$th generation segments to be oriented so that the positive direction is from the $(n-1)$ st generation end point to the $n$th generation end point, we define a chain $A_{1}$ on the linear graph which has the value

$$
-1 / 2 p(2 p-1)^{n-1}
$$


on each oriented segment of the $n$th generation. Now

$$
\partial A_{1}=\stackrel{0}{t_{0}}
$$

for at $t_{0}^{0}$ we have $2 p$ outgoing segments where the value of $A_{1}$ is $-1 /(2 p)$, while at the other points of the graph we have one incoming and $2 p-1$ outgoing segments with the value of the chain on the incoming segment being $-(2 p-1)$ times its value on each of the outgoing segments. Since there are $2 p(2 p-1)^{n-1}$ segments in the $n$th generation, we have

$$
\begin{aligned}
N\left(A_{1}\right) & =\sum_{n=1}^{\infty} 2 p(2 p-1)^{n-1}\left[2 p(2 p-1)^{n-1}\right]^{-2} \\
& =\sum_{n=1}^{\infty}\left[2 p(2 p-1)^{n-1}\right]^{-1} \\
& =\frac{2 p-1}{4 p(p-1)}
\end{aligned}
$$

if $p>1$. Thus in view of Proposition 28 we have the following proposition which has as a corollary an important result due to P. J. Myrberg [16].

Proposition 29. If $\tilde{W}$ is a Schottky covering surface of a compact surface whose genus is greater than one, then it is hyperbolic.

Corollary. The singular set of a Schottky group in the plane has positive capacity provided that the group has more than one generator.

31. A condition for capacity in the plane. We conclude this chapter by proving the following condition for capacity:

Proposition 30. Let $\Omega$ be a region in the plane containing the point $z=\infty$, and let $\left\{s_{i}^{2}\right\}$ be a triangulation of $\Omega$ with the property that there are only a finite number of the $s_{i}^{2}$ which are dissimilar in the sense of Euclidean geometry. In order that the complement of $\Omega$ have positive capacity it is sufficient that there be a cochain $A^{1}$ on $s_{i}^{2}$ with

$$
N\left(A^{1}\right)<\infty
$$

and

$$
\partial A^{1}=s_{0}^{2} .
$$

Proof. By virtue of the corollary to Theorem 11 it suffices to show that ${ }^{\cdot}$ the triangulation is uniform. But the $d z$-measure of two similar polygons is the same, as one can readily verify. Thus $\lambda_{d z}\left(s_{i}^{2}\right)$ has only a finite number of values and hence a finite upper bound. Consequently, the triangulation is uniform and the proposition is proved. 


\section{BIBLIOGRAPHY}

1. L. Ahlfors, Zur Theorie der Überlagerungsflächen, Acta Math. vol. 65 (1935).

2. - Über die Anwendung differentialgeoemtrische Methoden zur Untersuchen von Überlagerunsgflächen, Acta Societatis Scientiarum Fennicae II vol. 6 (1937).

3. — Z Zur Uniformisierungstheorie, Congrès des Mathématiques à Helsingfors, 1938.

4. - Die Begrunden des Dirichletschen Prinzips, Societas Scientiarum Fennica. Commentationes Physico-Mathematicae XI vol. 15 (1943).

5. - Normalintegrale auf offene Riemannschen Flächen, Annales Academiae Scientiarum Fennicae. Ser. A I no. 35 (1947).

6. - Open Riemann surfaces and extremal problems on compact subregions, Comment. Math. Helv. vol. 24 (1950).

7. - Remarks on the classification of open Riemann surfaces, Annales Academiae Scientiarum Fennicae. Ser. A I no. 87 (1951).

8. L. Ahlfors and A. Beurling, Invariants conformes et problèmes extrémaux, Comptes Rendus du Dixième Congrès Scandinave à Copenhague, 1946.

9. - Conformal invariants and function-theoretic null-sets, Acta Math. vol. 83 (1950).

10. R. Courant and D. Hilbert, Methoden der mathematischen Physik, vol. II, Berlin, 1937.

11. G. de Rham and K. Kodaira, Harmonic integrals, Institute for Advanced Study, Princeton, 1950.

12. K. Kodaira, Harmonic fields on Riemannian manifolds (generalized potential theory), Ann. of Math. vol. 50 (1949).

13. P. Laasonen, Zum Typenproblem der Riemannschen Flächen, Annales Academiae Scientiarum Fennicae. Ser. A I no. 11 (1942).

14. O. Lehto, On the existence of analytic functions with a finite Dirichlet integral, Annales Academiae Scientiarum Fennicae. Ser. A I no. 67 (1942).

15. P. J. Myrberg, Über die Existenz der Greenschen Funktione auf einer gegebenen Riemannschen Fläche, Acta Math. vol. 61 (1933).

16. - Die Kapazität der singularen Menge der linearen Gruppen, Annales Academiae Scientiarum Fennicae. Ser. A I no. 10 (1941).

17. - Über die analytische Fortsetzung von beschränkten Funktionen, Annales Academiae Scientiarum Fennicae. Ser. A I no. 58 (1949).

18. - Ü̈ber die Existenz von beschränktartigen automorphen Funktionen, Annales Academiae Scientiarum Fennicae. Ser. A I no. 77 (1950).

19. R. Nevanlinna, Eindeutige analytische Funktionen, Berlin, 1936.

20. - Quadratische integrierbare Differentiale auf einer Riemannschen Mannigfaltigkeit, Annales Academiae Scientiarum Fennicae. Ser. A I no. 1 (1941).

21. - Eindeutigkeitsfragen in der Theorie der konformen Abbildung, Comptes Rendus du Dixième Congrès Scandinave à Copenhague, 1946.

22. - Über das Anwachsen des Dirichletintegrals einer analytischen Funktionen auf einer offenen Riemannschen Flächen, Annales Academiae Scientiarum Fennicae. Ser. A I no. 45 (1948).

23. A. Pfluger, Über das Anwachsen eindeutiger analytischer Funktionen anf offenen Riemannschen Flächen, Annales Academiae Scientiarum Fennicae. Ser. A I no. 64 (1949).

24. H. L. Royden, Some remarks on open Riemann surfaces, Annales Academiae Scientiarum Fennicae. Ser. A I no. 85 (1951).

25. - Some counterexamples in the classification of open Riemann surfaces. To appear.

26. Leo Sario, Über Riemannschen Flächen mit hebbarem Rand, Annales Academiae Scientiarum Fennicae. Ser. A I no. 50 (1948).

27. —- Sur le problème du type des surface de Riemann, C.R. Acad. Sci. Paris vol. 229 (1949) pp. 1109-1111. 
28. - Existence des fonctions d'allure donnée sur une surface de Riemann arbitraire, C.R. Acad. Sci. Paris vol. 229 (1949) pp. 1293-1295.

29. - Quelques proprietés à la frontière se rattachant à la classification des surfaces de Riemann, C.R. Acad. Sci. Paris vol. 230 (1950) pp. 42-44.

30. - Existence des intégrales abéliennes sur les surfaces de Riemann arbitraires, C.R. Acad. Sci. Paris vol. 230 (1950) pp. 168-170.

31. - Questions d'existence au voisinage de la frontière d'une surface de Riemann, C.R. Acad. Sci. Paris vol. 230 (1950) pp. 269-271.

32. A. Speiser, Über Riemannschen Flächen, Comment. Math. Helv. vol. 4 (1932).

33. M. H. Stone, Linear transformations in Hilbert space and their applications to analysis, Amer. Math. Soc. Colloquium Publications, vol. 15, New York, 1932.

34. K. I. Virtanen, Über Abelsche Integrale auf nullberandeten Riemannschen Flächen von unendlichen Geschlecht, Annales Academiae Scientiarum Fennicae. Ser. A I no. 56 (1949).

35. - Über die Existenz von beschränkten harmonischen Funktionen auf offenen Riemannschen Flächen, Annales Academiae Scientiarum Fennicae. Ser. A I no. 75 (1950).

36. H. Wittich, Ein Kriterium zur Typenbestimmung von Riemannschen Flächen, Monatshefte für Mathematik und Physik vol. 44 (1936).

37. H. Weyl, Die Idee der Riemannschen Flächen, Berlin, 1923. (1940).

38. - The method of orthogonal projection in potential theory, Duke Math. J. vol. 7

\section{HARVARD UNIVERSITY, \\ Cambridge, Mass.}

